\title{
Gardnerella Vaginalis Alters Cervicovaginal Epithelial Cell Function Through Epithelial Cell-type Specific Immune Responses
}

Lauren Anton ( $\boldsymbol{\sim}$ lanton@pennmedicine.upenn.edu )

University of Pennsylvania

\section{Briana Ferguson}

University of Pennsylvania

Elliot S. Friedman

University of Pennsylvania

Kristin Gerson

University of Pennsylvania

Amy G. Brown

University of Pennsylvania

Michal A. Elovitz

University of Pennsylvania

\section{Research Article}

Keywords: cervix, lactobacillus crispatus, gardnerella vaginalis, epithelial barrier, inflammation, TLR2, preterm birth

Posted Date: January 14th, 2022

DOI: https://doi.org/10.21203/rs.3.rs-1251543/v1

License: (9) This work is licensed under a Creative Commons Attribution 4.0 International License. Read Full License 


\section{Abstract}

Background: The cervicovaginal (CV) microbiome is highly associated with vaginal health and disease in both pregnant and non-pregnant individuals. An overabundance of Gardnerella vaginalis in the CV space is commonly associated with adverse reproductive outcomes including bacterial vaginosis (BV), sexually transmitted diseases and preterm birth while the presence of Lactobacillus spp is often associated with reproductive health. While host-microbial interactions are hypothesized to contribute to $\mathrm{CV}$ health and disease, the mechanisms by which these interactions regulate $\mathrm{CV}$ epithelial function remain largely unknown.

Results: Using an in vitro co-culture model, we assessed the effects of Lactobacillus crispatus and G. vaginalis on the $\mathrm{CV}$ epithelial barrier, the immune mediators that could be contributing to decreased barrier integrity and the immune signaling pathways regulating the immune response. $G$. vaginalis, but not $L$. crispatus, significantly increased epithelial cell death and decreased epithelial barrier integrity in an epithelial cell-specific manner. A G. vaginalis-mediated epithelial immune response including NFkB activation and proinflammatory cytokine release was initiated partially through TLR2 dependent signaling pathways. Additionally, investigation of the cytokine immune profile in human CV fluid showed distinctive clustering of cytokines by $G$. vaginalis abundance and birth outcome.

Conclusions: The results of this study show both microbe- and epithelial cell-type specific effects on CV epithelial function. Altered epithelial barrier function through cell death and immune mediated mechanisms by $G$. vaginalis, but not $L$. crispatus, indicates that host epithelial cells respond to bacteriaassociated signals, resulting in altered epithelial function and ultimately CV disease. Additionally, distinct immune signatures associated with $G$. vaginalis or birth outcome provide further evidence that hostmicrobial interactions may contribute significantly to the biological mechanisms regulating reproductive outcomes.

\section{Introduction}

Host-microbe interactions play a significant role in the pathophysiology of human disease. Tissuespecific microbiomes are fundamental to host physiology and immunology. Interactions range from a mutual symbiosis (commensal) to disease-causing pathogenicity with many levels in between (contextual pathogen/pathobiont). Location-specific microbiomes have been shown to affect the host through multiple complex mechanisms including direct interactions with host cells, such as immune activation through bacterial cell well components or the release of microbial immune regulators including metabolites or anti-microbial proteins [1]. One of the most prominent and well-studied examples of hostmicrobe interactions occur at mucosal surfaces overlaying epithelial barriers, which can be found in specific human niches including the gut $[2,3]$. Crosstalk between microbiota and host epithelial cells can promote or diminish the health of the epithelial barrier causing disease states such as inflammatory bowel disease (IBD) [4-6]. 
Similarly, the composition and diversity of the vaginal microbiome has been shown to be highly associated with cervicovaginal (CV) health and disease [7]. The vaginal microbiome has been well characterized in both non-pregnant and pregnant individuals using high throughput 16S rRNA gene sequencing and at least five major community state types (CSTs) have been described [8-11]. Classification of CSTs has provided a systematic approach to studying the vaginal microbiome [8]. While there is variance in nomenclature, characterization of vaginal microbial communities is often defined by whether they are abundant or deficient in Lactobacillus spp. Consistent with this classification, four of these CSTs are dominated by Lactobacillus species including L. crispatus (CST-I), L. gasseri (CST-II), L. iners (CST-III) and L. jensenii (CST-V). CSTs dominated by Lactobacillus species are often indicative of a healthy vaginal space due to the production of high levels of lactic acid (resulting in low vaginal $\mathrm{pH}$ ) and bacteriocins produced by these bacteria $[12,13]$. In contrast, CST-IV is deplete of Lactobacillus species and is instead composed of mostly strict and facultative anaerobic bacteria, such as Gardnerella vaginosis, Mobiluncus, Atopobium vaginae, Bacterioides and Prevotellla. These microbes are typically associated with dysbiotic vaginal states such as bacterial vaginosis (BV) $[14,15]$, sexually transmitted diseases including human immunodeficiency virus (HIV) [16], and adverse reproductive outcomes [17, 18].

The vaginal microbiome has been associated with diverse aspects of female reproductive health including menstruation, menopause, infertility, and adverse pregnancy outcomes, specifically, spontaneous preterm birth (sPTB) $[7,19]$. sPTB, or delivery prior to 37 weeks of gestation, is a complex multi-factorial syndrome that remains poorly understood. While there are many factors that are associated with the development of SPTB, over the past 10 years, several studies have identified associations between SPTB and the CV microbiome providing evidence that a Lactobacillus-deficient vaginal microbiome could be a significant mechanistic contributor to the development of SPTB [9-11, 20, 21]. Data from a large, nested case-control study from our group showed significant associations between seven bacteria taxa, including M. curtsii/mulieris, Atopobium, and Megasphaera (all CST-IV), and increased risk of sPTB [10]. The risk of SPTB was positively associated with abundance of these specific taxa. [8]. Even though a Lactobacillus-deficient CST-IV state may not be associated with a clear pathological phenotype, the presence of CST-IV confers a higher risk of adverse vaginal pathologies such as herpes papillomavirus (HPV), BV, HIV and other Sexually transmitted diseases [22-25]. Understanding the mechanisms by which Lactobacillus-deficient microbial communities impart an increased risk of adverse reproductive outcomes remains an obstacle to advancing reproductive health. Therefore, elucidating the host-microbe interactions within the CV space becomes necessary for targeted therapeutics to prevent and/or treat CV-based adverse reproductive outcomes such as BV, STIs, and sPTB.

sPTB is one of the most common adverse reproductive outcomes. While the essential mechanisms driving SPTB remain elusive, recent research ascribes a crucial role for the cervical epithelial barrier in SPTB. Breakdown of the cervical epithelial barrier is hypothesized to be an initiating step in the cervical remodeling process that occurs prior to delivery [26-29]. Multiple molecular factors have been shown to alter cervical epithelial barrier integrity including inflammatory mediators [30], cellular adhesion 
proteins [29-32] , and miRNAs [29, 31, 33-35]. We propose that the CV microbiome is a common regulator of these molecular pathways. Previous studies published by our laboratory and others have shown that bacteria-free supernatants from $G$. vaginalis and $M$. mulieris cause breakdown of the ectocervical and endocervical epithelial barriers through cleavage of adhesion proteins, immune activation, and epigenetic regulation $[28,29]$. Furthermore, mouse studies have shown that vaginal colonization with $G$. vaginalis results in SPTB [36]. While the results of these studies provide evidence that alterations in the CV microbiome can significantly alter epithelial cell function, they did not investigate the mechanistic drivers of host-microbe epithelial interactions.

In vivo, host-microbial interactions in the CV space are complex and likely driven by microbes, microbial by-products, and their communication with specific immune and epithelial cell types. As vaginal and cervical epithelial cells originated from different embryological origins [37-39], they likely have distinct cellular roles with complex cell specific functions. Despite their unique origins, very few studies have investigated how host-microbe effects differ between cervical and vaginal cells [40, 41]. In vivo, both live bacteria and the molecules they secrete (e.g., proteins, metabolites, extracellular vesicles) may elicit relevant cellular effects, and it is unknown how these effects might be similar or divergent across CV epithelial cells. Therefore, revealing the interactions between bacteria and their by-products with different epithelial cell types within the CV space are necessary to advance our understanding of host-microbial interactions and their contributions to reproductive health and disease.

Therefore, the objectives of this study were 1) to determine the effects of live CV bacteria and their supernatants on the integrity of the cervical and vaginal epithelial barrier; 2) to elucidate the immune profile resulting from interactions between live bacteria or their supernatants and host CV epithelial cells; 3 ) to determine if toll-like receptor (TLR) signaling pathways are necessary for the activation of the epithelial cell immune responses from common CV microbes; and 4) to validate in vitro findings by assessing immune signatures in the CV space of pregnant individuals with a high abundance of $G$. vaginalis.

\section{Materials And Methods Cell Culture}

Ectocervical (Ect/E6E7, AATC\# CRL-2614) (Ecto), endocervical (End1/E6E7, AATC\# CRL-2615) (Endo) and vaginal (VK2/E6E7, ATCC\# CRL-2616) (VK2) human epithelial cell lines (American Type Culture Collection, Manassas, VA) were cultured in Keratinocyte-Serum Free Media (K-SFM) supplemented with $0.1 \mathrm{ng} / \mathrm{mL}$ epidermal growth factor and $50 \mathrm{ug} / \mathrm{mL}$ bovine pituitary extract (Gibco, Life Technologies), 100 $\mathrm{U} / \mathrm{mL}$ penicillin, and $100 \mu \mathrm{g} / \mathrm{mL}$ of streptomycin at $37^{\circ} \mathrm{C}$ in a $5 \% \mathrm{CO}_{2}$ humidified incubator.

TLR2 (NF-kB-SEAP / KI-IL-8 Lucia) dual-reporter human embryonic kidney (HEK) 293 cells (Invivogen, San Diego, CA), a TLR2 reporter cell line, expresses the human TLR2 gene, an NF-kB/AP1-inducible SEAP (secreted embryonic alkaline phosphatase) reporter gene and the Lucia luciferase reporter gene under the 
control of the endogenous IL-8 promoter. These cells also show no activity of TLR3, TLR5, and TNFR (Tumor Necrosis Factor receptor). The HEK TLR2 cells were cultured according to the manufacture's protocol. Briefly, HEK-TLR2 cells were grown in Dulbecco's Modified Eagle's Medium (DMEM, Mediatech, Corning, Glendale, AZ) containing $4.5 \mathrm{~g} / \mathrm{L}$ glucose, $2 \mathrm{mM} \mathrm{L}$-glutamine, 10\% heat-inactivated fetal bovine serum (FBS, 30 mins at $56^{\circ} \mathrm{C}$ ), $100 \mathrm{ug} / \mathrm{mL}$ Normocin (Invivogen) and selective antibiotics $-100 \mathrm{ug} / \mathrm{mL}$ Hygromycin B Gold (Invivogen) and $50 \mathrm{ug} / \mathrm{mL}$ Zeocin (Invivogen). Cells were grown in 100mm culture dishes at $37^{\circ} \mathrm{C}$ in a $5 \% \mathrm{CO}_{2}$ humidified incubator.

\section{Bacterial Cultures and Preparation of Bacteria-Free Supernatants}

Bacterial strains, Lactobacillus crispatus (ATCC 33197) or Gardnerella vaginalis (ATCC 14018), were obtained from the American Type Culture Collection (Manassas, VA). G. vaginalis was grown on Human Blood Tween Bilayer agar plates (Fisher Scientific) and L. crispatus was grown on De Man, Rogosa and Sharpe agar (Fisher Scientific); both strains were grown in New York City (NYC) III broth. Bacteria were grown at $37^{\circ} \mathrm{C}$ in an anaerobic glove box (Coy Labs, Grass Lake, MI).

For each experiment the following bacterial growth protocol was followed: L. crispatus and G. vaginalis glycerol stocks were streaked on agar plates and grown overnight. Individual colonies were used to inoculate starter cultures and grown overnight. Starter cultures were diluted to an optical density of 0.2 and then used to inoculate working cultures, which were grown for 20 hours prior to use in experiments. Bacterial densities of the working cultures were estimated the day of the experiment based on optical density readings at $600 \mathrm{~nm}$ using an Epoch2 plate reader (Biotek, Winooski, VT), and the appropriate volume was centrifuged at 13,000 $\mathrm{g}$ for $3 \mathrm{~min}$. The bacterial pellets were resuspended in K-SFM cell culture media without antibiotics and added to cells at $10^{4}-10^{6} \mathrm{CFUs} /$ well. Precise bacterial densities of the working culture were determined by CFU assays (plating serial dilutions of the working cultures). For all experiments, reported bacterial densities are $+/-0.5 \log$ of the noted bacterial density (CFU/well).

To obtain supernatants, the working cultures were centrifuged at $13,000 \mathrm{~g}$ for $3 \mathrm{~min}$ and the supernatant was filtered through a $0.22 \mu \mathrm{m}$ filter (Fisher Scientific) to remove any remaining live bacteria. Bacteria-free supernatants are diluted to $10 \% \mathrm{v} / \mathrm{v}$ in K-SFM cell culture media without antibiotics.

\section{Epithelial Cell/ Bacteria Co-Culture In vitro Model}

Ectocervical, endocervical and vaginal cells were plated at $2.0 \times 10^{5}$ cells/well in twenty-four well plates containing K-SFM without antibiotics. The next day, the cells were treated with either live L. crispatus or $G$. vaginalis $\left(1 \times 10^{4}-1 \times 10^{6} \mathrm{CFU} /\right.$ well) or $10 \%(\mathrm{v} / \mathrm{v})$ bacteria-free supernatants $\left(1 \times 10^{5}-1 \times 10^{7} \mathrm{CFU} / \mathrm{mL}\right.$ culture density) in K-SFM cell growth media for 24 hours. For cells treated with bacteria-free supernatants from $L$. crispatus, K-SFM media was supplemented with $50 \mathrm{mM}$ HEPES and sodium bicarbonate $(3000 \mathrm{mg} / \mathrm{L}$ total 
concentration) to bring the $\mathrm{pH}$ of the media up to a physiological level (7.2) as L. crispatus bacteria produces high amounts of lactic acid during growth. Without $\mathrm{pH}$ adjustments, even at lower volume per volume percentages, the cells did not survive. In additional experiments, ectocervical, endocervical and vaginal cells were pre-treated with a neutralizing IgA monoclonal antibody to human TLR2 $(10 \mathrm{ug} / \mathrm{mL})$ (anti-hTLR2-IgA, InvivoGen, San Diego, CA) for one hour prior to exposure to live bacteria or supernatants from $L$. crispatus or $G$. vaginalis. For all supernatant experiments, cells were also treated with NYCIII bacterial growth media alone as a negative control to determine any baseline effects of the growth media on the outcome of interest. At the end of each experiment, cell culture media was collected for cell death, ELISA assays and/or the cells were collected in Trizol (Invitrogen, Thermo-Fisher Scientific) for RNA extraction.

\section{Differential Interference Contrast Imaging}

Ectocervical, endocervical and vaginal epithelial cells were plated at $2.0 \times 10^{5}$ cells/dish on $35 \mathrm{~mm}$ high glass bottom $\mu$-dishes (ibidi, Martinsried, Germany) coated with $0.1 \%$ gelatin for $24 \mathrm{hrs}$. Live L. crispatus and $G$. vaginalis were added to the cells and incubated 4-6 hours prior to imaging. Differential Interference Contrast (DIC) images of our epithelial cell / bacteria co-culture with L. crispatus and G. vaginalis were taken using the Zeiss Axio Observer 7 Widefield microscope using the 100x objective (Zeiss 100x/1.4 NA oil Plan-Apochromat) with ZEN Blue software (version 2.5).

\section{HEK-hTLR2 Treatments and NFkB and IL-8 Detection}

HEK-hTLR2 cells were plated at $7.5 \times 10^{4}$ cells/well in 96 well plates containing DMEM $+10 \%$ heatinactivated FBS without antibiotics. The next day, the cells were treated with either live L. crispatus or $G$. vaginalis $\left(10^{4}-10^{6} \mathrm{CFU} /\right.$ well) or $10 \%(\mathrm{v} / \mathrm{v})$ bacteria-free supernatants $\left(10^{7}-10^{5} \mathrm{CFU} / \mathrm{mL}\right.$ culture density) in DMEM cell culture media for 24 hours. In additional experiments, the cells were pre-treated with the TLR2 neutralizing antibody, anti-hTLR2-IgA (InvivoGen) for one hour prior to exposure to live bacteria or supernatants. In these experiments, the TLR2 agonist FSL-1 $(10 \mathrm{ng} / \mathrm{mL}$, Sigma-Aldrich, St. Louis, MO) was used as a positive control for antibody efficacy. For detection of a nuclear factor kappa-B (NFkB) response (SEAP reporter), cell culture supernatants were incubated with Quanti-Blue solution (Invivogen) for 1 hour, pictures were taken of the plate, and absorbance was read at 630nm on a SpectraMax i3x plate reader (Molecular Devices). For detection of an IL-8 response (Lucia luciferase reporter), cell culture supernatants from the same experiment were combined with Quanti-Luc solution (Invivogen) and luminescence was read immediately on a SpectraMax i3X plate reader. Additionally, cell culture supernatants were used in cell death assays as described below.

\section{Cell Death Assay}


Ectocervical, endocervical, vaginal and HEK-hTLR2 cells were grown as described above. The release of lactate dehydrogenase (LDH) from ectocervical, endocervical, and vaginal cells ( $n=3-9$ independent experiments per cell type) was measured using the CytoTox 96 Non-radioactive cytotoxicity assay (Promega, Madison, WI). This assay allows for the quantitative measurement of LDH that is released upon cell lysis using a coupled enzymatic assay that results in the conversion of a tetrazolium salt into a red formazan product. The amount of color formed is proportional to the amount of LDH released as the cells lyse. Color formation was read on a colorimetric plate reader at $490 \mathrm{~nm}$ and absorbance values were recorded.

\section{Cell Permeability Experiments}

Ectocervical, endocervical and vaginal cell permeability was determined using an In Vitro Vascular Permeability Assay (Millipore, Bedford, MA). Briefly, ectocervical, endocervical and vaginal cells were plated at $1.0 \times 10^{6}$ cells $/ \mathrm{mL}$ into 24 well hanging cell culture inserts which contain $1 \mu \mathrm{m}$ pores with a transparent polyethylene terephthlate (PET) membrane pre-coated with collagen. The cells were treated with either live L. crispatus or $G$. vaginalis $\left(10^{6}-10^{4} \mathrm{CFU} /\right.$ well) or $10 \%(\mathrm{v} / \mathrm{v})$ bacteria-free supernatants from $L$. crispatus $(n=6)$ and $G$. vaginalis $(n=9)$ for 24 hours. In additional experiments, ectocervical, endocervical and vaginal cells were pre-treated with the anti-hTLR2 antibody prior to exposure to live bacteria or supernatants from L. crispatus or G. vaginalis (as described above) for 24 hours $(n=9)$. For all supernatant experiments, cells were also treated with NYCIII bacterial growth media alone as a negative control to determine any baseline effects of the growth media on cell permeability. After 24 hours of treatment, the media was removed and phenol red free K-SFM media (ScienCell Laboratories, Carlsbad,

CA) containing FITC-Dextran was added to the top of the insert. The movement of FITC-Dextran from the top insert to the bottom was measured after two hours by a fluorescent plate reader at $485 \mathrm{~nm}$ and $535 \mathrm{~nm}$, excitation and emission, respectively.

\section{ELISA}

Ectocervical, endocervical and vaginal cells were cultured in 24-well plates and treated with either live bacteria or bacteria-free supernatants as stated above. IL-8 was measured in cell culture media after 24 hours of treatment. The expression of IL-8 was measured by a ligand-specific commercially available ELISA kit that utilizes a quantitative sandwich enzyme immunoassay technique using regents from R\&D Systems (Minneapolis, MN).

\section{Luminex Assay}

Ectocervical, endocervical and vaginal cells were cultured and treated with live bacteria or bacteria-free supernatants as stated above. A 41-plex cytokine/chemokine (HCYTMAG-60K-PX41) and a TGFB 3-plex (TGFBMAG-64K-03) human magnetic bead Luminex panel (EMD Millipore, Billerica, MA) was run on 1) 
ectocervical, endocervical and vaginal cell culture media after 24 hours of treatment with L. crispatus $(n=3)$ or $G$. vaginalis $(n=3)$ live bacteria or bacteria-free supernatants and 2$)$ cell culture media from ectocervical, endocervical and vaginal cells pre-treated with an anti-TLR2 blocking antibody followed by L. crispatus $(n=3)$ or $G$. vaginalis $(n=3)$ live bacteria and 3 ) human cervical vaginal fluid (CVF) collected from individuals with or without a SPTB with low or high CV G. vaginalis abundance. Human CVF was collected using Dacron swabs (Starplex, ThermoFisher). Materials on the swabs were eluted in sterile PBS with a protease inhibitor cocktail (Complete Mini) for $5 \mathrm{~min}$ to release the soluble proteins. All samples were run in duplicate, per the manufacturer's protocol on the FLEXMAP3D Luminex platform (Luminex, Austin, TX). Absolute quantification in $\mathrm{pg} / \mathrm{mL}$ was obtained using a standard curve generated by a fiveparameter logistic (5PL) curve fit using Xponent 4.2 software (Luminex). Fold change values were calculated between treatment groups and the non-treated control (NTC) for live bacteria or between the treatment groups and the NYC media control for bacteria-free supernatants. For those cells pre-treated with the anti-TLR2 blocking antibody, fold change was calculated between the bacteria treatment alone and the bacteria plus the anti-TLR2 antibody. For human samples, data is expressed as average $\mathrm{pg} / \mathrm{mL}$. Heatmaps were created using R.

\section{mRNA Isolation from Epithelial Cells}

Following treatment with live L. crispatus and G. vaginalis, ectocervical, endocervical and vaginal cells were collected in Trizol (Invitrogen, Thermo-Fisher Scientific) and underwent phenol-chloroform extraction. The resulting aqueous phase was further column purified with the miRNeasy kit (Qiagen, Hilden, Germany) according to the manufacturer's protocol for total RNA isolation. RNA concentration was determined via a NanoDrop 2000 Spectrophotometer (Nanodrop ${ }^{\mathrm{TM}}$ Rockland, DE) prior to the generation of cDNA.

\section{cDNA generation and qPCR}

cDNA was generated from $1 \mu \mathrm{g}$ of isolated RNA from ectocervical, endocervical and vaginal cells using the high capacity cDNA reverse transcription kit (Applied Biosystems, Thermo-Fisher Scientific). qPCR was performed on the 7900HT Real-Time PCR System (Applied Biosystems) using the TaqMan Universal PCR Master Mix (Applied Biosystems) according to the manufacturers' protocols. The standard curve method was used for relative expression quantification using the RQ manager software v2.4 (Applied Biosystems). The relative abundance of the target of interest was divided by the relative abundance of $18 \mathrm{~S}$ in each sample to generate a standardized abundance for the target transcript of interest. All mRNA primers were purchased from Applied Biosystems: TLR2, MYD88, NOD1, NOD2 and 18S.

\section{Human Samples}


To investigate if the cytokine signature in pregnant individuals with high and low G. vaginalis abundance was different, we utilized CVF samples from a prospective cohort study of 2000 individuals with singleton pregnancies. Details of this study are available in the primary report [10]. All participants provided written informed consent and the study was approved by the Institutional Review Board at the University of Pennsylvania (IRB \#818914) and the University of Maryland School of Medicine (HP00045398). Cervicovaginal specimens were self-collected by the participant or collected by a research coordinator between 16 and 20 weeks. A set of cervicovaginal swabs was obtained including an ESwab (COPAN) stored in $1 \mathrm{~mL}$ of Amies Transport Medium and a Dacron swab stored without buffer. All samples were immediately frozen at $-80^{\circ} \mathrm{C}$ until processing. All delivery outcomes were recorded. Cases of PTB were adjudicated by the PI (Elovitz) to determine if they were spontaneous as previously described [10]. To assess the CV microbial communities, 16s rRNA gene amplification and sequence was performed as described previously [10].

For this study, a nested case-control selection of subjects was performed (Table 1). Inclusion criteria were individuals identified as being in CST IV at 16-20 weeks by microbial sequencing $(n=131)$. From this cohort of individuals with CST IV, four groups were identified by birth outcome and $G$. vaginalis abundance. The four groups were 1) Term with low $G$. vaginalis (GV abundance lower than 0.2 ), 2) SPTB with low $G$. vaginalis, 3) Term with high $G$. vaginalis (GV abundance of 0.2 or greater) or 4) sPTB with high $G$. vaginalis. $G$. vaginalis abundance cutoff of 0.2 was determined based on the calculated median from the whole cohort.

\section{Table 1}

\section{Patient Demographics}

\begin{tabular}{|c|c|c|c|c|c|}
\hline \multirow[t]{2}{*}{ Maternal characteristics } & All & & \multirow[t]{2}{*}{$\begin{array}{l}\text { Term low } \\
\text { GV }\end{array}$} & \multirow{2}{*}{$\begin{array}{l}\text { sPTB } \\
\text { high GV }\end{array}$} & \multirow[t]{2}{*}{$\begin{array}{l}\text { Term } \\
\text { High GV }\end{array}$} \\
\hline & $(n=131)$ & $\begin{array}{l}\text { low } \\
\text { GV }\end{array}$ & & & \\
\hline
\end{tabular}

Race

0.138

\begin{tabular}{cllllll}
\hline White & $12(9.2)$ & $1(4.4)$ & $3(6.5)$ & $4(20.0)$ & $4(9.5)$ & \\
\hline Black & 118 & 22 & $43(93.5)$ & $15(75.0)$ & $38(90.5)$ & \\
& $(90.1)$ & $(95.7)$ & & & & \\
\multicolumn{1}{c}{ Other } & $1(0.8)$ & $0(0.0)$ & $0(0.0)$ & $1(5.0)$ & $0(0.0)$ & \\
\hline Age (Mean, SD) & 26.6 & 27.7 & 26.6 & $26.3(6.1)$ & $26.2(5.8)$ & $0.76^{*}$ \\
& $(5.8)$ & $(5.7)$ & $(5.7)$ & & & \\
\hline $\begin{array}{l}\text { Gestational age at delivery } \\
\text { (Mean, SD) }\end{array}$ & 36.0 & 27.8 & 39.2 & $30.9(6.3)$ & $39.4(0.8)$ & $<0.001^{*}$ \\
\hline
\end{tabular}

Presented as $\mathrm{n}$ (col \%); Chi-square p-value unless otherwise indicated. *Kruskal-Wallis test sPTB - spontaneous preterm birth, GV - Gardnerella vaginalis 


\section{Statistical Analysis}

Statistical analyses were performed for all experiments with the GraphPad Prism Software (Version 9.0, San Diego, CA). For data that were normally distributed, one-way analysis of variance (ANOVA) was performed. If statistical significance was reached $(p<0.05)$, then pair-wise comparison with a Tukey post hoc test was performed for multiple comparisons. If data were not normally distributed, then the nonparametric Kruskal-Wallis test was used and pairwise comparison was done using Dunn's multiple comparison test. Analysis of Luminex cytokine data was performed using a one-way ANOVA followed by different post hoc tests comparing treatment groups to 1) a non-treated control (NTC) was done using a Dunnett's multiple comparison test or 2) between select pairs of treatment groups was done using Sidak's multiple comparisons test. Human CVF Luminex data were analyzed by two tailed t-test with term delivery compared to SPTB in both the low and high $G$. vaginalis abundance groups. If the variances between groups were significantly different, then a t-test with Welch's correction was used.

\section{Results}

\section{G. vaginalis co-localizes with cervicovaginal epithelial cells resulting in increased cell death in a host-microbial co- culture model}

In creating our in vitro host-microbial co-culture model, characterization of bacterial interactions with host epithelial cells was necessary before moving forward with subsequent experiments. Differential Interference Contrast (DIC) images of L. crispatus and G. vaginalis co-cultured with ectocervical (Figure $1 A, B$ ), endocervical (Figure $1 C, D$ ) and vaginal (Figure 1E, F) cells showed limited co-localization between the epithelial cells and L. crispatus (Figure 1A, C, E). However, there was significant colocalization/interaction between the CV epithelial cells and G. vaginalis (Figure 1B, D, F). Co-culture of $G$. vaginalis with all three cell types resulted in observed cell blebbing indicative of cellular stress and death. Cytotoxicity assays confirmed the observed cell death in all three cell lines (Figure 1G, H, I). Lactate dehydrogenase (LDH) was increased in a dose-dependent manner $\left(10^{4}-10^{6} \mathrm{CFU} /\right.$ well) after co-culture with G. vaginalis (but not L. crispatus) in ectocervical $(p<0.0001)$, endocervical $(p<0.0001)$ and vaginal $(p<0.0001)$ cells.

\section{L. crispatus and G. vaginalis have divergent effects on cervical and vaginal epithelial barrier permeability}

To determine if the presence of live L. crispatus or G. vaginalis or their supernatants alone are essential for functional alteration of the cervical and/or vaginal epithelial cell barriers, cell permeability assays were performed in ectocervical, endocervical, and vaginal epithelial cells. Live G. vaginalis significantly increased cell permeability in a dose-dependent manner in all three cell lines (ecto: $p<0.0001$, endo: 
$p<0.001$, vaginal: $p<0.0001$, with no significant effects seen with live $L$. crispatus at the same doses (Figure 2A, C, E). CV cells co-cultured with live $G$. vaginalis had significantly higher cell permeability when compared to those co-cultured with L. crispatus at both the $10^{6}$ (ecto: $p<0.0001$, endo: $p=0.0072$, vaginal: $p<0.0001$ ) and $10^{5}$ (ecto: $p<0.0001$, endo: $p=0.0233$, vaginal: $p<0.0001$ ) doses of bacteria but not at $10^{4}$ (CFU/well). Exposure of ectocervical, endocervical, and vaginal cells to bacteria-free supernatants from $G$. vaginalis had no effect on cell permeability at any of the doses tested, while L. crispatus supernatants significantly decreased cell permeability, when compared to the NYC control, similarly at all three doses tested (ecto: $p<0.0001$, endo: NS, vaginal: $p<0.0001$ ) (Figure 2B, D, F). There was no significant effect of the bacterial growth media alone (NYC control) on cell permeability of ectocervical, endocervical, or vaginal cells.

\section{G. vaginalis and $L$. crispatus bacteria and their bacterial-free supernatants induce distinct immune profiles in a CV epithelial cell-type specific manner}

We have previously shown that $G$. vaginalis bacteria-free supernatants can increase the release of a varied group of inflammatory mediators from ectocervical cells [29]; however, the contribution of live bacteria (including bacterial cell wall and its associated proteins) to activating a host-epithelial cell immune response was not assessed. Therefore, we sought to examine the effects of both live bacteria and bacteria-free supernatants on the activation of the immune response from all three CV epithelial cell lines. Of the 44 cytokines/chemokines included in the discovery-based Luminex arrays (41-plex immune and 3-plex TGFB), 28 were detectable in at least one of the three cell lines after exposure to live bacteria (Figure 3A), while 27 were detectable after exposure to bacteria-free supernatants (Figure 3B). A full list of all cytokines/chemokines included in the Luminex assays and their average values $(\mathrm{pg} / \mathrm{mL})$ in each cell type can be found in Supplemental Table 1A (live bacteria) and 1B (bacteria-free supernatants). Most of the detectable cytokines were significantly increased after exposure to live $G$. vaginalis versus exposure to $L$. crispatus (when each are compared to NTC) (Supplemental Table 2). PDGF-AA was the only cytokine decreased after live $G$. vaginalis exposure in all three cell lines. Very few analytes were altered by live L. crispatus with two, three, and four cytokines changed in ectocervical, endocervical, and vaginal cells, respectively (Supplemental Table 2A). In cells exposed to bacteria-free supernatants, there were significant numbers of cytokines altered in both the L. crispatus and G. vaginalis groups. In cells exposed to bacteria-free supernatants from L. crispatus, the majority of altered cytokines were decreased, while in cells exposed to $G$. vaginalis, the majority of altered cytokines were increased (Supplemental Table 2B). Overall, similar cytokines/chemokines were detected after co-culture with either live bacteria or bacteriafree supernatants; however, the levels of those cytokines varied widely between the two exposures with 13 cytokines being higher after exposure to live bacteria when compared to supernatants. Nine were lower after live bacteria exposure compared to supernatants, and five were similar. A representative subset of cytokines (Figure 3C, D) demonstrates the differential cell-specific responses of ectocervical, 
endocervical, and vaginal cells to either live bacteria (Figure 3C) or bacteria-free supernatants (Figure 3D).

\section{L. crispatus and G. vaginalis bind to TLR2 activating NFkB}

To identify the intracellular immune pathways being activated by $G$. vaginalis we utilized HEK TLR2 reporter cells. Live G. vaginalis and L. crispatus induced NFkB activation in a dose-dependent manner with $G$. vaginalis-exposed cells sustaining a higher level (than L. crispatus) of NFkB across all three bacterial doses tested (Figure 4A, B). IL-8 activation was significantly elevated in a dose-dependent manner to live $G$. vaginalis but not $L$. crispatus (except at the $10^{6}$ dose) (Figure $4 C$ ). A similar response was seen after exposure to bacteria-free supernatants from $G$. vaginalis and L. crispatus (Figure $4 \mathrm{E}$ G). HEK TLR2 cell death was similar to that seen in the CV epithelial cells with a dose-dependent increase in LDH in live $G$. vaginalis co-cultures and no significant difference found after exposure to live $L$. crispatus nor any of the bacteria-free supernatants (Figure 4D, H).

\section{G. vaginalis-mediates increased cytokine release partially through TLR2 activated signaling pathways}

While this study has shown that NFkB and a multitude of cytokines are activated/increased in response to live $G$. vaginalis, at least partially, through activation of the TLR2 receptor, we have not investigated if the TLR2 receptor is a necessary activation signal in the CV immune response. TLR2, MYD88, NOD1, and NOD2 are all expressed in ectocervical, endocervical, and vaginal epithelial cell lines (Figure 5A). The expression of TLR2 ( $p=0.0007$ versus endo, $p<0.0001$ versus vaginal) and MYD88 ( $<<0.0001$ versus endo, $p<0.0001$ versus vaginal) are significantly higher in ectocervical cells when compared to endocervical and vaginal cell lines. Both NOD1 and NOD2 were equally expressed across cell lines. To determine if TLR2 activation is essential for the epithelial immune response and consequent cytokine increase, we blocked the TLR2 receptor and examined cytokine output after exposure to L. crispatus and G. vaginalis in both HEK TLR2 cells and all three CV cell lines. In HEK TLR2 cells, FSL (a potent TLR2 agonist, $p<0.0001), L$. crispatus $(\mathrm{p}<0.0001)$ and $G$. vaginalis $(\mathrm{p}<0.0001)$ - mediated increases in NFkB were significantly reduced with TLR2 blockade (Figure 5B). Similarly, pretreatment with the anti-TLR2 antibody also reduced IL-8 activation after exposure to FSL $(\mathrm{p}<0.0001)$ and $G$. vaginalis $(\mathrm{p}=0.0007)$ but not $L$. crispatus (Figure $5 C)$. Preliminary investigation into the $G$. vaginalis-mediated increase in cytokine expression in CV cell lines showed that blocking TLR2 also significantly reduced IL-8 expression in a cell-type specific manner: $17 \%$ reduction in ectocervical cells ( $p=0.0067$, Figure $5 D), 66 \%$ reduction in endocervical cells $(p<0.0001$, Figure $5 \mathrm{E}$ ), and $37 \%$ reduction in vaginal cells ( $<<0.0001$, Figure $5 \mathrm{~F}$ ).

To further investigate the role of TLR2 activation in G. vaginalis-mediated cytokine production in all the CV cell lines, we assessed the immune response after TLR2 blockade using the same Luminex panels as described above (Figure 6A, B, C). As we observed previously, there was a varied immune response 
between the three cell lines with ectocervical cells exhibiting the greatest overall number of cytokines altered after TLR2 blockade in both L. crispatus and G. vaginalis exposed cells followed by endocervical and then vaginal cells (Supplemental Table 3). TLR2 blockade inhibited the $G$. vaginalis-induced increase in cytokine expression across all cell lines, except for IL-1RA which was increased. In contrast, TLR2 blockade only mitigated the L. crispatus-induced cytokine expression in ectocervical but not endocervical or vaginal cells (Figure 6). Interestingly, TLR2 blockade also increased seven L. crispatus-induced cytokines in ectocervical cells and one cytokine in vaginal cells.

\section{Activation of TLR2 is sufficient, but not essential, for the $G$. vaginalis-mediated breakdown of the cervical and vaginal epithelial barrier}

Since we have evidence that live $G$. vaginalis induces a significant immune response through the activation of TLR2, we wanted to determine if the TLR2 receptor is an essential mechanism in the observed loss of integrity of the cervical and vaginal epithelial barrier. Blocking the TLR2 receptor in ectocervical (Figure 7A), endocervical (Figure 7B) and vaginal (Figure 7C) epithelial cells did not mitigate the ability of live $G$. vaginalis to breakdown the epithelial barrier at either of the two doses of $G$. vaginalis tested.

\section{Pregnant individuals with a high CV G. vaginalis abundance who deliver preterm have a distinct CV immune signature from those with a term birth}

To investigate if $G$. vaginalis induces a distinct immune signature in those who ultimately have a SPTB versus term birth, we performed Luminex on CVF from 131 pregnant individuals categorized into CST-IV by $16 \mathrm{~S}$ rRNA gene sequencing [10]. Four groups comprised of individuals with high or low $G$. vaginalis CVF abundance and term or SPTB delivery outcomes were analyzed. Secondarily, the CVF immune profile of individuals with high $G$. vaginalis abundance and a SPTB was compared against the immune profile our in vitro co-culture model (CV epithelial cells exposed to live $G$. vaginalis). All 44 cytokines studied were detectable in human CVF. Of those 44 , seven were significantly $(p<0.05)$ increased in individuals with high CVF G. vaginalis abundance who underwent a preterm delivery compared to those that delivered at term (Figure 8A). Six additional cytokines (EGF, G-CSF, IL-13, MIP-1a, TNF-a, SCD40L) were non-significantly increased $(p<0.10)$ in those who had a SPTB and high $G$. vaginalis abundance. Four cytokines were significantly decreased $(p<0.05)$ in CVF from individuals with a low $G$. vaginalis abundance who had a sPTB compared to those with a term birth (Figure 8A). Of the 20 cytokines that were significantly increased in the G. vaginalis epithelial cell co-culture model (Figure 3A), six cytokines overlapped between human CVF from individuals with high $G$. vaginalis and a SPTB and CV epithelial cells exposed to live $G$. vaginalis (Figure $8 \mathrm{~B}$ and $8 \mathrm{C}$ ). 


\section{Discussion}

This study provides evidence that CV bacteria have significant microbe- and cell specific effects on host epithelial cell function indicating that complex host-microbe interactions contribute to vaginal health. We have shown that $G$. vaginalis, a vaginal microbe commonly associated with adverse reproductive health, results in both functional and immune-based host responses as evidenced by increased CV epithelial cell death, epithelial barrier breakdown and immune activation partially through TLR2/NFkB induced expression of chemokines/cytokines. However, L. crispatus, a microbe associated with optimal vaginal health, does not induce changes in epithelial cell death nor epithelial barrier function. Unique to this work, we reveal that vaginal and cervical epithelial cells possess different immune responses to similar microbe challenges and thus emphasize the need to understand the complexity of the CV space for optimizing reproductive health. Importantly, these studies demonstrate that activation of TLR2 is a shared pathway across epithelial cell types in response to $G$. vaginalis exposure. Results from our human study confirm our in vitro findings that the host-microbe mediated $\mathrm{CV}$ immune response is associated with the abundance of select microbes and notably that an increased cytokine response is associated with birth outcome.

In both pregnant and non-pregnant individuals, the primary function of the cervical and vaginal epithelial cells is to create a barrier against harmful microbes. To accomplish this, multiple diverse mechanisms are used including the creation of a physical barrier made up of the epithelial cells themselves, the expression of adhesion and tight junction proteins responsible for holding the cells tightly together, and the production of a mucous layer that protects the epithelial cells by entrapping or killing pathogens. Pathogenic microbes have historically developed ways to circumvent this barrier, as is the case with $G$. vaginalis,which expresses a number of factors that enhance its virulence potential including adherence to CV epithelial cells, biofilm forming capabilities, mucous degradation (salidase)[42], and host cell cytotoxicity[43]. G. vaginalis has been shown to produce vaginolysin[44, 45], a cholesterol dependent cytolysin that acts as cytotoxic pore forming toxin and, consequently, kills host epithelial cells. Similar to previous studies investigating the cytotoxic effects of $G$. vaginalis[46], we observed CV cell death in our in vitro host-microbial co-culture model providing evidence that $G$. vaginalis functional outcomes were conserved in this model. Interestingly, the cytotoxic effect of $G$. vaginalis was similar across all three epithelial cell types with a slightly more potent effect in ectocervical versus endocervical and vaginal cells. L. crispatus, as expected, had no effect on CV epithelial cell death despite being exposed to a large dose $\left(10^{6} \mathrm{CFU} /\right.$ well $)$ of bacteria.

The integrity of the CV barrier is essential not only to vaginal health (vaginal epithelial barrier), but also to a successful pregnancy (cervical epithelial barrier). The breakdown of the cervical epithelial barrier is thought to be a critical step in the cervical remodeling and dilation process that occurs prior to the onset of labor[47]. Consequently, early disruption of the epithelial barrier is hypothesized to contribute to preterm delivery through premature cervical remodeling or microbe-associated inflammatory mechanisms. A previous study from our laboratory supports this hypothesis as it demonstrated that intravaginal $G$. vaginalis colonization in a pregnant mouse model results in preterm birth with an 
activated immune response, breakdown of the epithelial barrier, induced cervical remodeling, and altered cervical biomechanics[32]. Consistent with our previous findings in an animal model, we show that live $G$. vaginalis decreases epithelial barrier integrity in vitro in all three $\mathrm{CV}$ cell types. We have previously demonstrated that $G$. vaginalis bacteria-free supernatants (factors secreted by bacteria) are also able to breakdown the cervical epithelial barrier[29]. In contrast to this prior report, in our current study, we found that bacteria-free supernatants did not have the same effect on epithelial cell permeability. One notable difference is the exposure time used in this study (24 hours) versus our previous study (48 hours). While exposure time may be a cause of the difference in findings with bacteria-free supernatants, it is also worth considering the more potent effects of live bacteria on epithelial cell function (predominantly increased cell death) as this likely occurs in vivo. While there was a significant difference in both ectocervical and vaginal (but not endocervical) cell permeability between G. vaginalis and L. crispatus bacteria-free supernatant exposed cells, the effect of $G$. vaginalis supernatants was diminished compared to live $G$. vaginalis. These results do provide evidence that live bacteria (and their cell wall) may be a more effective or significant contributor to epithelial barrier function than bacterial secreted factors alone. However, a key finding of these experiments is that microbes and their supernatants elicit different biological effects on CV epithelial cells. In vivo, both the live bacteria and their secreted factors likely contribute to the $\mathrm{CV}$ host response. Therefore, to fully understand the biological pathways that govern vaginal/reproductive health, it will be essential to reveal the harmful and beneficial effects of both live bacteria and their supernatants. As such, further investigation into the composition (e.g., proteins, metabolites, etc.) of bacteria-free supernatants would be warranted to fully understand their role in regulating $\mathrm{CV}$ epithelial function.

A breakdown in the CV barrier likely has multiple overlapping causes. Therefore, while epithelial cell death definitively plays a significant role in decreased barrier integrity, there are other mechanisms that have been shown to contribute to a breakdown in the cervical and vaginal epithelial barriers including inflammation[30]. As part of the epithelial barrier, one of the main functions of epithelial cells is to initiate an innate immune response to fight invading pathogens. Several previous studies from our laboratory and others have shown that $G$. vaginalis (live and supernatants) induces a robust but widely varied and complex inflammatory response in CV epithelial cells[29, 48, 49]. Similarly, in this study, the presence of live bacteria revealed a microbe- and cell-specific immune profile. $G$. vaginalis exposure resulted in the activation of an immune response across all three $\mathrm{CV}$ epithelial cells lines, however, the level of cytokine production across the three cell types was often diverse and varied by each cytokine analyzed. At a macroscopic level, vaginal epithelial cells seemed to have a more robust innate response to live $G$. vaginalis when compared to the cervical epithelial cells. This finding suggests that vaginal epithelial cells may act as a first line of defense against pathogens entering the vaginal canal and may help to protect the cervix from infection, especially during pregnancy. The immune response evoked by $G$. vaginalis bacteria-free supernatants was also robust in activating cytokines, and like our previous published study[29], L. crispatus also significantly altered cytokine production albeit mostly in a downward direction indicative of its commensal, and perhaps protective, functions. These results indicate that CV epithelial cells can recognize multiple different bacterial components, either from bacterial cell wall or secreted 
factors, and mount an essential immune response. We hypothesized that this type of redundancy makes it more difficult for harmful bacteria to evade host immune recognition.

Epithelial cell recognition of bacterial components has been shown to activate the innate immune response through toll like receptor (TLR) signaling. Of the TLRs, TLR2 is known to be activated bypathogen-associated molecular patterns (PAMPs)includinglipoproteins which are ubiquitous to all bacteria and highly expressed in the outer membrane of gram-positive bacteria. Once activated, TLR2 initiates intracellular signalingpathways which induce nuclear translocation of NFkB to modulate gene transcription and consequent inflammatory cytokine productionand immune cell infiltration[50, 51].Previous studies have shown that $C V$ lavage samples from clinical cases of $B V$ upregulate cytokines through TLR2-mediated mechanisms $[52,53]$. There is a paucity of data regarding the role of TLR signaling in the setting of CV host-microbe interactions. Using a TLR2 reporter cell line, we found that exposure to live and bacteria-free supernatants of G. vaginalis significantly induced NFkB and IL-8. Interestingly, L. crispatus also activated NFkB but that activation did not result in increased IL-8, except at the highest bacterial inoculum. These results indicate that high doses of bacteria, independent of bacteria species, activate a TLR2-mediated immune response. The non-specific activation of TLR2 by assumed healthy and non-healthy microbes may be a regulatory mechanism by the $\mathrm{CV}$ epithelium to prevent bacterial overgrowth. Alternatively, it is possible that a secondary signal or that a threshold of NFkB activation may be needed to activate cytokine production in response to L. crispatus or other CV microbes. The ability of $G$. vaginalis to cause host immune activation at a dose where $L$. crispatus does not suggest that host cells are able to recognize bacteria specific PAMPs and mount an immune response to more pathogenic bacteria. Additionally, like our cell death and permeability results, live bacteria exposure, compared to bacterial supernatants, results in a more robust IL-8 response, again indicating that whole bacteria have more substantial effects on these cellular functions than bacterial secreted factors alone.

It is well known that immune system activation is a redundant process with the ability of many different signals/receptors to be activated resulting in the same outcome of upregulated cytokines which also have overlapping functions (cytokine pleiotropy)[54]. Therefore, identifying which factors within the immune response are essential for altering CV epithelial cell function become necessary to target these pathways for future therapeutic or preventative strategies against conditions associated with non-optimal microbial communities in the CV space such as BV, STI acquisition, or SPTB. Since we have shown in this study that CV epithelial cells express TLR2 and the adaptor protein, MYD88, needed for NFkB activation[51, 55, 56],we investigated if TLR2 activation is an essential mechanism for bacteria-induced host immune activation. After blocking the TLR2 receptor, the reduction in NFkB activation seen with both live $L$. crispatus and $G$. vaginalis exposure also resulted in a mitigated IL-8 response. The complete mitigation of L. crispatus-induced NFkB and IL-8, but not G. vaginalis, indicates that TLR2 may not be the only immune pathway activated by $G$. vaginalis. Interestingly, in CV epithelial cells, blocking TLR2 resulted in varying levels of IL-8 reduction depending on the cell type. The biggest reduction in IL-8 (66\% after blocking TLR2) was seen in endocervical cells. This result agrees with the results of our Luminex assay, where the greatest number of $G$. vaginalis-induced cytokines were reduced after blocking TLR2 in 
endocervical cells perhaps indicating that TLR2 signaling is a bigger contributor to the immune response in endocervical cells than the other cell types. Of note, all three CV cell types express NOD1 and NOD2 which recognize intracellular PAMPs that can enter the cell through phagocytosis or membrane pores (such as those created by vaginolysin). NODs are part of the NLRP3 inflammasome which can activate NFkB signaling independently of membrane bound TLRs. G. vaginalis has been shown to activate the NLRP3 inflammasome in macrophages and monocytes $[57,58]$. Therefore, it is possible that NOD signaling may be a significant regulator of $G$. vaginalis-mediated inflammation in CV cells, especially in ectocervical and vaginal cells where TLR2 is likely not the only mediator of host-microbial mediated inflammation.

While the TLR2 blockade inhibited G.vaginalis-mediated epithelial immune activation, it did not prevent G. vaginalis-induced changes in epithelial cell permeability. Our data suggest that while TLR2 receptor blockade prevents some immune response, it is insufficient to limit the diverse downstream molecular effects from exposure to G.vaginalis. Consistent with the redundancy of the host immune response, it is plausible that multiple pathways need to be blocked to significantly mitigate the $G$. vaginalis-mediated breakdown of the CV epithelial barrier. In addition to inflammation, other biological mechanisms are known to regulate the epithelial barrier including cell death and tight junctions, therefore, it is quite likely that a combination of interconnected factors are necessary for a functional change to the barrier. Future research targeting a combination of biological mechanisms would be warranted to fully assess the essential mechanisms regulating $\mathrm{CV}$ epithelial barrier function.

We acknowledge that an in vitro model of host-microbial interactions has its limitations mostly due to the nature of a single cell type acting alone without the effects of surrounding cells including stromal cells and immune effector cells (neutrophils, T-cells, NK cells, macrophages). Therefore, we chose to compare our in vitro cytokine results with those seen in a well characterized human cohort of CVF samples collected from pregnant individuals characterized as having CST-IV, with high or low G. vaginalis abundance, who ultimately had a term delivery or SPTB[10]. Although human data inherently has wide variability, the immune signature revealed a distinctive clustering of cytokines within each group analyzed. Interestingly, there seems to be a larger number of elevated cytokines in CVF from pregnant individuals with low compared to high $G$. vaginalis abundance early in pregnancy. Since all individuals in this study have a CST-IV microbial state, these results would indicate that the interaction of select CST-IV microbes (and not $G$. vaginalisalone), within the community and/or the metabolic output of the community, play an important role in regulating the $\mathrm{CV}$ immune response. Historically, studies investigating $G$. vaginalis and the immune response mostly in vaginal samples from individuals with BV have found significant increases in inflammatory cytokines includingIL-8, IL-6, IL-1a and IL-13[59-61].In recent years, there has been some debate as to whether $G$. vaginalis initiates a significant inflammatory response, especially in cases of asymptomatic BV[62]. It has been suggested that $G$. vaginalis in combination with other CST-IV or other BV associated bacteria (Atopium vaginae and Snethia spp) are needed to cause significant clinical or inflammatory symptoms $[62,63]$. Part of this discrepancy could also be due to the identification of more/less virulent strains (biotypes/ecotypes) of G. vaginaliswhich are associated with BV status and symptoms $[64,65]$. In this cohort, since we did not categorize patients by 
BV status, but by GV abundance and delivery outcome instead, it is difficult to determine if the $G$. vaginalis strain subtypes present in these individuals fall into the more virulent category. However, it is interesting to note that when comparing individuals with high $G$. vaginalis abundance who had a term versus SPTB, there is a cluster of elevated cytokines (EGF, G-CSF, GM-CSF, IL-2, PDGF, Eotaxin and IL-9) that may give some insight into identifying those individuals who are at higher risk for SPTB. A more indepth analysis of this data including correlations with patient metadata, other bacterial species abundance and immune regulators is planned in future studies.

In comparing the immune signature from our human dataset to that of our in vitro host-microbial coculture model, we found both similarities and differences in the baseline presence and elevation of the cytokines. Not surprisingly, there were more detectable cytokines in human cervicovaginal samples when compared to our in vitro cultures (44 vs 28 cytokines). Additionally, there was some overlap in elevated cytokines between the high $G$. vaginalis abundance with SPTB group and our CV cells exposed to live $G$. vaginalis (six cytokines) indicating that our in vitro co-culture partially mimics the activated immune response seen in human samples. Of note, IL-8 was not increased in the high $G$. vaginalis abundance group in our human cohort. While this was surprising given the strong correlation observed with IL-8 and G. vaginalis exposure in our in vitro cultures, we acknowledge that the inflammatory profiles between a single cell culture and a true physiological response will be different. However, the primary outcome of a G. vaginalis-mediated inflammatory response is similar in both sample subsets. Given this result, the in vitro co-culture model remains a useful tool in studying the mechanisms regulating host-microbial interactions with the knowledge that findings using this model should be verified in human samples as well.

\section{Conclusion}

Overall, the results of this study have provided critical insight into the host-microbial interactions between CV epithelial cells and the microbiota that inhabit the CV space. This study has shown that G. vaginalis alters cervical and vaginal epithelial cell function by decreasing epithelial barrier integrity, increasing cell death, and activating an inflammatory response that is partially regulated by TLR2/NFkB-based signaling pathways. Importantly, this study shows distinct epithelial cell-specific immune responses to microbespecific signals that reveal complex interactions within the CV space. Understanding differences in the epithelial immune response in the cervix versus vagina will also provide insights into cervical or vaginal specific immune-based therapies/treatments for known G. vaginalis-associated states such as BV, sexually transmitted diseases (e.g., HIV), or adverse pregnancy outcomes including preterm birth. Additionally, differential immune profiling of CST-IV dominant pregnant individuals with altered levels of $G$. vaginalis abundance and delivery outcomes provides a distinct immune signature for risk stratification of individuals who may ultimately have a SPTB. The results from this study begin to ascribe a biological mechanism as to how host epithelial cells and CV microbes interact to regulate cellular function in both commensal and dysbiotic states. 


\section{Abbreviations}

CV - cervicovaginal space

CST - community state type

BV - bacterial vaginosis

HIV - human immunodeficiency virus

sPTB - spontaneous preterm birth

TLR - toll-like receptor

Ecto - ectocervical epithelial cells

Endo - endocervical epithelial cells

VK2 - vaginal epithelial cells

K-SFM - keratinocyte serum-free media

HEK - human embryonic kidney cells

NYC - New York City III bacterial growth media

LDH - lactate dehydrogenase

\section{Declarations}

\section{Ethical Approval and Consent to Participate}

All participants provided written informed consent and the study was approved by the Institutional Review Board at the University of Pennsylvania (IRB \#818914) and the University of Maryland School of Medicine (HP-00045398).

\section{Consent for Publication}

Not Applicable

\section{Availability of data and materials}

All data generated or analyzed during this study are included in this published article and its supplementary information files. 


\section{Competing Interests}

MAE receives salary support from NIH (NIAID, NINR, and NICHD). She is a consultant for MIRVIE. ESF has consulted for Astarte Medical Partners and Enzymetrics Bioscience, Inc.

\section{Funding}

Support for this research was provided by the National Institutes of Health (NIH) National Institute of Child Health and Human Development (NICHD) (1R01HD102318 and 5R01HD098867) and March of Dimes Prematurity Research Center at the University of Pennsylvania (22-FY18-812).

\section{Author Contributions}

LA wrote the manuscript and created the figures. LA, AGB, ESF and MAE conceived and designed the experiments. LA, BF, KSG, ESF performed the experiments. LA analyzed the data. All authors contributed to manuscript revision and approved the final manuscript.

\section{Acknowledgements}

The authors would like to acknowledge the Microbial Culture and Metabolomics Core of the PennCHOP Microbiome Program, specifically Dillon Murphy, for providing the microbial culture services for these studies. Additionally, we acknowledge the Human Immunology Core (P30-CA016520) at the University of Pennsylvania for their help with all the Luminex assays performed in this study.

\section{Author's Information}

None

\section{References}

1. Kogut $\mathrm{MH}$, Lee A, Santin E. Microbiome and pathogen interaction with the immune system. Poultry Science.2020; 99:1906-1913.

2. Okumura R, Takeda K. Maintenance of intestinal homeostasis by mucosal barriers. Inflammation and Regeneration.2018; 38:5.

3. Allaire JM, Crowley SM, Law HT, Chang SY, Ko HJ, Vallance BA. The intestinal epithelium: Central coordinator of mucosal immunity. Trends Immunol.2018; 39:677-696.

4. Frank DN, St Amand AL, Feldman RA, Boedeker EC, Harpaz N, Pace NR. Molecular-phylogenetic characterization of microbial community imbalances in human inflammatory bowel diseases. 
Proceedings of the National Academy of Sciences of the United States of America.2007; 104:1378013785.

5. Kiely CJ, Pavli P, O'Brien CL. The role of inflammation in temporal shifts in the inflammatory bowel disease mucosal microbiome. Gut Microbes.2018; 9:477-485.

6. Caparrós E, Wiest R, Scharl M, Rogler G, Gutiérrez Casbas A, Yilmaz B, Wawrzyniak M, Francés R. Dysbiotic microbiota interactions in crohn's disease. Gut Microbes.2021; 13:1949096.

7. Kroon SJ, Ravel J, Huston WM. Cervicovaginal microbiota, women's health, and reproductive outcomes. Fertil Steril.2018; 110:327-336.

8. Ravel J, Gajer P, Abdo Z, Schneider GM, Koenig SSK, McCulle SL, Karlebach S, Gorle R, Russell J, Tacket CO, et al. Vaginal microbiome of reproductive-age women. Proceedings of the National Academy of Sciences of the United States of America.2011; 108 Suppl 1:4680-4687.

9. Romero R, Hassan SS, Gajer P, Tarca AL, Fadrosh DW, Bieda J, Chaemsaithong P, Miranda J, Chaiworapongsa T, Ravel J. The vaginal microbiota of pregnant women who subsequently have spontaneous preterm labor and delivery and those with a normal delivery at term. Microbiome.2014; 2:18.

10. Elovitz MA, Gajer P, Riis V, Brown AG, Humphrys MS, Holm JB, Ravel J. Cervicovaginal microbiota and local immune response modulate the risk of spontaneous preterm delivery. Nat Commun.2019; 10:1305-1305.

11. Fettweis JM, Serrano MG, Brooks JP, Edwards DJ, Girerd PH, Parikh HI, Huang B, Arodz TJ, Edupuganti L, Glascock AL, et al. The vaginal microbiome and preterm birth. Nat Med.2019; 25:10121021.

12. Tyssen D, Wang YY, Hayward JA, Agius PA, DeLong K, Aldunate M, Ravel J, Moench TR, Cone RA, Tachedjian G. Anti-hiv-1 activity of lactic acid in human cervicovaginal fluid. mSphere.2018; 3.

13. Stoyancheva G, Marzotto M, Dellaglio F, Torriani S. Bacteriocin production and gene sequencing analysis from vaginal lactobacillus strains. Arch Microbiol.2014; 196:645-653.

14. Morrill S, Gilbert NM, Lewis AL. Gardnerella vaginalis as a cause of bacterial vaginosis: Appraisal of the evidence from in vivo models. Front Cell Infect Microbiol.2020; 10:168.

15. Schwebke JR, Muzny CA, Josey WE. Role of gardnerella vaginalis in the pathogenesis of bacterial vaginosis: A conceptual model. J Infect Dis.2014; 210:338-343.

16. Hashemi FB, Ghassemi M, Roebuck KA, Spear GT. Activation of human immunodeficiency virus type 1 expression by gardnerella vaginalis. J Infect Dis.1999; 179:924-930.

17. Holst E, Goffeng AR, Andersch B. Bacterial vaginosis and vaginal microorganisms in idiopathic premature labor and association with pregnancy outcome. J Clin Microbiol.1994; 32:176-186.

18. Bretelle F, Rozenberg P, Pascal A, Favre R, Bohec C, Loundou A, Senat MV, Aissi G, Lesavre N, Brunet J, et al. High atopobium vaginae and gardnerella vaginalis vaginal loads are associated with preterm birth. Clin Infect Dis.2015; 60:860-867. 
19. Ma B, Forney LJ, Ravel J. Vaginal microbiome: Rethinking health and disease. Annu Rev Microbiol.2012; 66:371-389.

20. DiGiulio DB, Callahan BJ, McMurdie PJ, Costello EK, Lyell DJ, Robaczewska A, Sun CL, Goltsman DSA, Wong RJ, Shaw G, et al. Temporal and spatial variation of the human microbiota during pregnancy. Proceedings of the National Academy of Sciences.2015; 112:11060-11065.

21. Callahan BJ, DiGiulio DB, Goltsman DSA, Sun CL, Costello EK, Jeganathan P, Biggio JR, Wong RJ, Druzin ML, Shaw GM, et al. Replication and refinement of a vaginal microbial signature of preterm birth in two racially distinct cohorts of us women. Proceedings of the National Academy of Sciences.2017; 114:9966-9971.

22. Gosmann C, Anahtar MN, Handley SA, Farcasanu M, Abu-Ali G, Bowman BA, Padavattan N, Desai C, Droit L, Moodley A, et al. Lactobacillus-deficient cervicovaginal bacterial communities are associated with increased hiv acquisition in young south african women. Immunity.2017; 46:29-37.

23. Edwards Vonetta L, Smith Steven B, McComb Elias J, Tamarelle J, Ma B, Humphrys Michael S, Gajer P, Gwilliam K, Schaefer Alison M, Lai Samuel K, et al. The cervicovaginal microbiota-host interaction modulates chlamydia trachomatis infection. mBio.10:e01548-01519.

24. Anahtar MN, Byrne EH, Doherty KE, Bowman BA, Yamamoto HS, Soumillon M, Padavattan N, Ismail N, Moodley A, Sabatini ME, et al. Cervicovaginal bacteria are a major modulator of host inflammatory responses in the female genital tract. Immunity.2015; 42:965-976.

25. Dareng EO, Ma B, Adebamowo SN, Famooto A, Ravel J, Pharoah PP, Adebamowo CA. Vaginal microbiota diversity and paucity of lactobacillus species are associated with persistent hrhpv infection in hiv negative but not in hiv positive women. Sci Rep.2020; 10:19095.

26. Timmons BC, Mitchell SM, Gilpin C, Mahendroo MS. Dynamic changes in the cervical epithelial tight junction complex and differentiation occur during cervical ripening and parturition. Endocrinology.2007; 148:1278-1287.

27. Nallasamy S, Mahendroo M. Distinct roles of cervical epithelia and stroma in pregnancy and parturition. Semin Reprod Med.2017; 35:190-200.

28. Dude CM, Saylany A, Brown A, Elovitz M, Anton L. Microbial supernatants from mobiluncus mulieris, a bacteria strongly associated with spontaneous preterm birth, disrupts the cervical epithelial barrier through inflammatory and mirna mediated mechanisms. Anaerobe.2020; 61:102127.

29. Anton L, Sierra L-J, DeVine A, Barila G, Heiser L, Brown AG, Elovitz MA. Common cervicovaginal microbial supernatants alter cervical epithelial function: Mechanisms by which lactobacillus crispatus contributes to cervical health. Frontiers in Microbiology.2018; 9.

30. Nold C, Anton L, Brown A, Elovitz M. Inflammation promotes a cytokine response and disrupts the cervical epithelial barrier: A possible mechanism of premature cervical remodeling and preterm birth. Am J Obstet Gynecol.2012; 206:208.e201-207.

31. Anton L, DeVine A, Sierra L-J, Brown AG, Elovitz MA. Mir-143 and mir-145 disrupt the cervical epithelial barrier through dysregulation of cell adhesion, apoptosis and proliferation. Scientific reports.2017; 7:3020-3020. 
32. Sierra LJ, Brown AG, Barilá GO, Anton L, Barnum CE, Shetye SS, Soslowsky LJ, Elovitz MA. Colonization of the cervicovaginal space with gardnerella vaginalis leads to local inflammation and cervical remodeling in pregnant mice. PLoS One.2018; 13:e0191524.

33. Elovitz MA, Anton L, Bastek J, Brown AG. Can microrna profiling in maternal blood identify women at risk for preterm birth? American Journal of Obstetrics and Gynecology.2015; 212:782.e781-782.e785.

34. Elovitz MA, Brown AG, Anton L, Gilstrop M, Heiser L, Bastek J. Distinct cervical microrna profiles are present in women destined to have a preterm birth. American Journal of Obstetrics and Gynecology.2014; 210:221.e221-221.e211.

35. Winger EE, Reed JL, Ji X, Gomez-Lopez N, Pacora P, Romero R. Micrornas isolated from peripheral blood in the first trimester predict spontaneous preterm birth. PLOS ONE.2020; 15:e0236805.

36. Sierra L-J, Brown AG, Barilá GO, Anton L, Barnum CE, Shetye SS, Soslowsky LJ, Elovitz MA. Colonization of the cervicovaginal space with gardnerella vaginalis leads to local inflammation and cervical remodeling in pregnant mice. PLOS ONE.2018; 13:e0191524.

37. Forsberg J-G. Cervicovaginal epithelium: Its origin and development. American Journal of Obstetrics and Gynecology.1973; 115:1025-1043.

38. Fritsch H, Hoermann R, Bitsche M, Pechriggl E, Reich O. Development of epithelial and mesenchymal regionalization of the human fetal utero-vaginal anlagen. Journal of Anatomy.2013; 222:462-472.

39. Reich $\mathrm{O}$, Fritsch $\mathrm{H}$. The developmental origin of cervical and vaginal epithelium and their clinical consequences: A systematic review. J Low Genit Tract Dis.2014; 18:358-360.

40. Trifonova RT, Doncel GF, Fichorova RN. Polyanionic microbicides modify toll-like receptor-mediated cervicovaginal immune responses. Antimicrob Agents Chemother.2009; 53:1490-1500.

41. McGowin CL, Ma L, Martin DH, Pyles RB. Mycoplasma genitalium-encoded mg309 activates nfkappab via toll-like receptors 2 and 6 to elicit proinflammatory cytokine secretion from human genital epithelial cells. Infect Immun.2009; 77:1175-1181.

42. Lewis WG, Robinson LS, Gilbert NM, Perry JC, Lewis AL. Degradation, foraging, and depletion of mucus sialoglycans by the vagina-adapted actinobacterium gardnerella vaginalis. J Biol Chem.2013; 288:12067-12079.

43. Patterson JL, Stull-Lane A, Girerd PH, Jefferson KK. Analysis of adherence, biofilm formation and cytotoxicity suggests a greater virulence potential of gardnerella vaginalis relative to other bacterialvaginosis-associated anaerobes. Microbiology (Reading).2010; 156:392-399.

44. Gelber SE, Aguilar JL, Lewis KL, Ratner AJ. Functional and phylogenetic characterization of vaginolysin, the human-specific cytolysin from gardnerella vaginalis. J Bacteriol.2008; 190:38963903.

45. Lawrence SL, Gorman MA, Feil SC, Mulhern TD, Kuiper MJ, Ratner AJ, Tweten RK, Morton CJ, Parker MW. Structural basis for receptor recognition by the human cd59-responsive cholesterol-dependent cytolysins. Structure.2016; 24:1488-1498.

46. Randis TM, Zaklama J, LaRocca TJ, Los FC, Lewis EL, Desai P, Rampersaud R, Amaral FE, Ratner AJ. Vaginolysin drives epithelial ultrastructural responses to gardnerella vaginalis. Infect Immun.2013; 
$81: 4544-4550$.

47. Timmons $B$, Akins M, Mahendroo M. Cervical remodeling during pregnancy and parturition. Trends Endocrinol Metab.2010; 21:353-361.

48. Garcia EM, Kraskauskiene V, Koblinski JE, Jefferson KK, Young VB. Interaction of gardnerella vaginalis and vaginolysin with the apical versus basolateral face of a three-dimensional model of vaginal epithelium. Infection and Immunity.2019; 87:e00646-00618.

49. Eade CR, Diaz C, Wood MP, Anastos K, Patterson BK, Gupta P, Cole AL, Cole AM. Identification and characterization of bacterial vaginosis-associated pathogens using a comprehensive cervicalvaginal epithelial coculture assay. PLOS ONE.2012; 7:e50106.

50. Yang RB, Mark MR, Gurney AL, Godowski PJ. Signaling events induced by lipopolysaccharideactivated toll-like receptor 2 . J Immunol.1999; 163:639-643.

51. Häcker H, Vabulas RM, Takeuchi O, Hoshino K, Akira S, Wagner H. Immune cell activation by bacterial cpg-DNA through myeloid differentiation marker 88 and tumor necrosis factor receptor-associated factor (traf)6. Journal of Experimental Medicine.2000; 192:595-600.

52. Mares D, Simoes JA, Novak RM, Spear GT. TIr2-mediated cell stimulation in bacterial vaginosis. J Reprod Immunol.2008; 77:91-99.

53. Zariffard MR, Novak RM, Lurain N, Sha BE, Graham P, Spear GT. Induction of tumor necrosis factoralpha secretion and toll-like receptor 2 and 4 mrna expression by genital mucosal fluids from women with bacterial vaginosis. J Infect Dis.2005; 191:1913-1921.

54. Ozaki K, Leonard WJ. Cytokine and cytokine receptor pleiotropy and redundancy*. Journal of Biological Chemistry.2002; 277:29355-29358.

55. Fan J, Frey RS, Malik AB. TIr4 signaling induces tlr2 expression in endothelial cells via neutrophil nadph oxidase. J Clin Invest.2003; 112:1234-1243.

56. Schnare M, Barton GM, Holt AC, Takeda K, Akira S, Medzhitov R. Toll-like receptors control activation of adaptive immune responses. Nature Immunology.2001; 2:947-950.

57. Vick EJ, Park HS, Huff KA, Brooks KM, Farone AL, Farone MB. Gardnerella vaginalis triggers nlrp3 inflammasome recruitment in thp-1 monocytes. J Reprod Immunol.2014; 106:67-75.

58. Xiang N, Yin T, Chen T. Gardnerella vaginalis induces nlrp3 inflammasome-mediated pyroptosis in macrophages and thp-1 monocytes. Exp Ther Med.2021; 22:1174.

59. Jespers V, Kyongo J, Joseph S, Hardy L, Cools P, Crucitti T, Mwaura M, Ndayisaba G, Delany-Moretlwe $\mathrm{S}$, Buyze J, et al. A longitudinal analysis of the vaginal microbiota and vaginal immune mediators in women from sub-saharan africa. Sci Rep.2017; 7:11974.

60. Hedges SR, Barrientes F, Desmond RA, Schwebke JR. Local and systemic cytokine levels in relation to changes in vaginal flora. J Infect Dis.2006; 193:556-562.

61. Kyongo JK, Crucitti T, Menten J, Hardy L, Cools P, Michiels J, Delany-Moretlwe S, Mwaura M, Ndayisaba G, Joseph S, et al. Cross-sectional analysis of selected genital tract immunological 
markers and molecular vaginal microbiota in sub-saharan african women, with relevance to hiv risk and prevention. Clin Vaccine Immunol.2015; 22:526-538.

62. Muzny CA, Laniewski P, Schwebke JR, Herbst-Kralovetz MM. Host-vaginal microbiota interactions in the pathogenesis of bacterial vaginosis. Current Opinion in Infectious Diseases.2020; 33.

63. Hinderfeld AS, Phukan N, Bär A-K, Roberton AM, Simoes-Barbosa A, Herbert DBR. Cooperative interactions between $<i>$ trichomonas vaginalis $</ i>$ and associated bacteria enhance paracellular permeability of the cervicovaginal epithelium by dysregulating tight junctions. Infection and Immunity.2019; 87:e00141-00119.

64. Cornejo OE, Hickey RJ, Suzuki H, Forney LJ. Focusing the diversity of gardnerella vaginalis through the lens of ecotypes. Evol Appl.2018; 11:312-324.

65. Castro J, Alves P, Sousa C, Cereija T, França Â, Jefferson KK, Cerca N. Using an in-vitro biofilm model to assess the virulence potential of bacterial vaginosis or non-bacterial vaginosis gardnerella vaginalis isolates. Scientific reports.2015; 5:11640-11640.

\section{Figures}


A.

\section{Ectocervical cells}

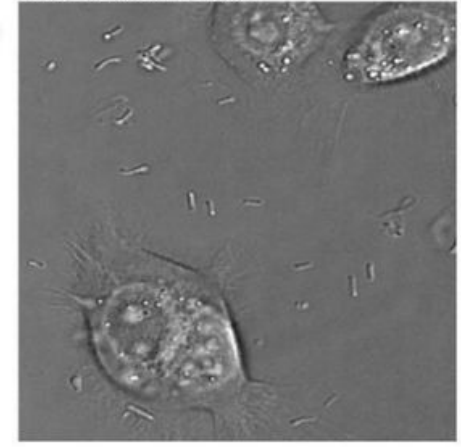

Endocervical cells

C.

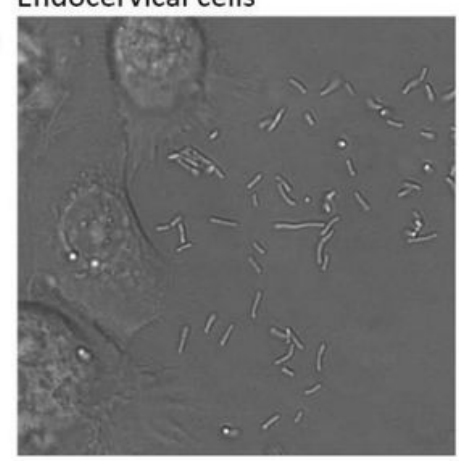

E.

Vaginal cells

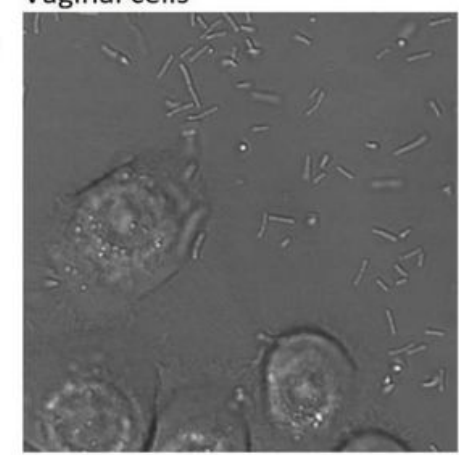

L. crispatus
B.

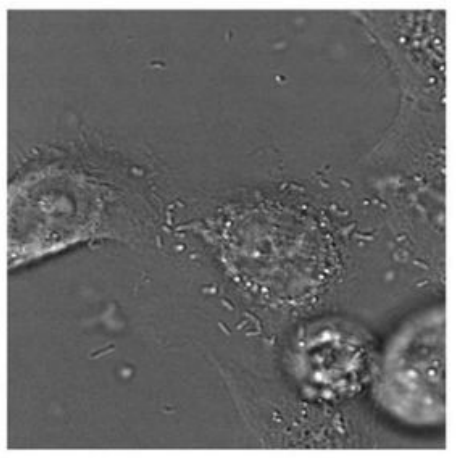

D.

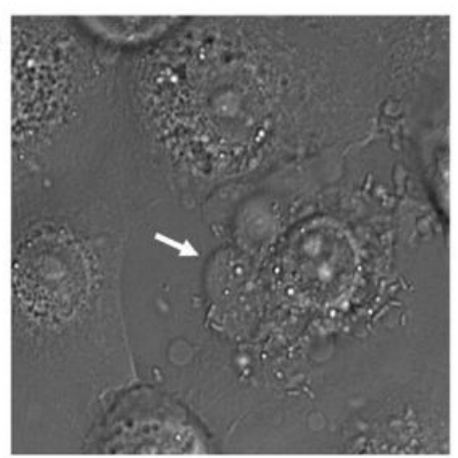

F.

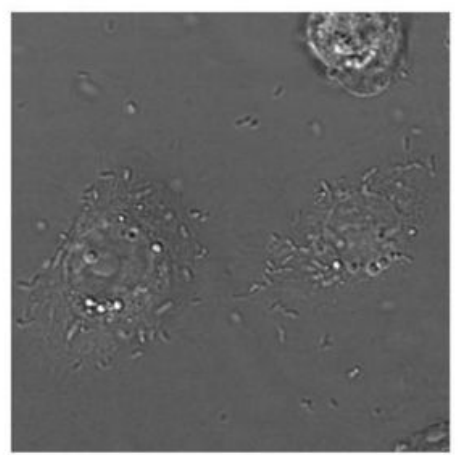

G. vaginalis
G.

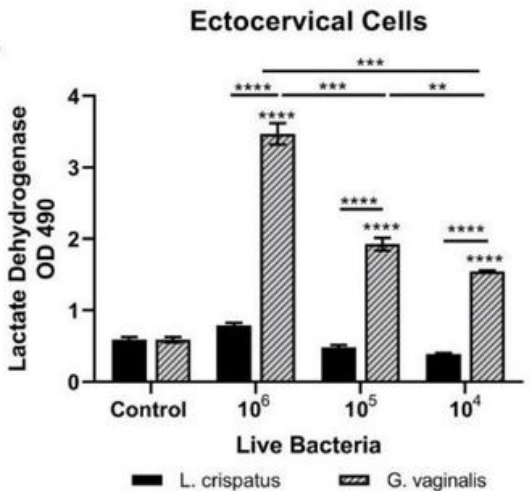

$\mathrm{H}$.

Endocervical Cells

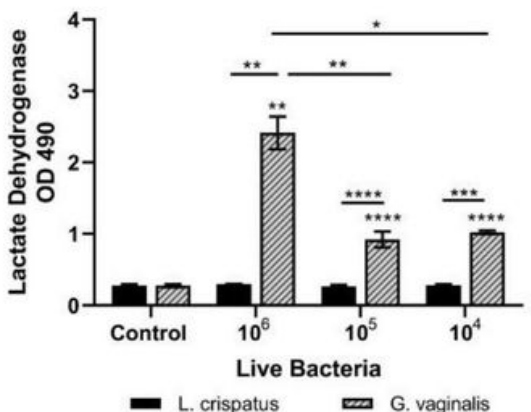

I.

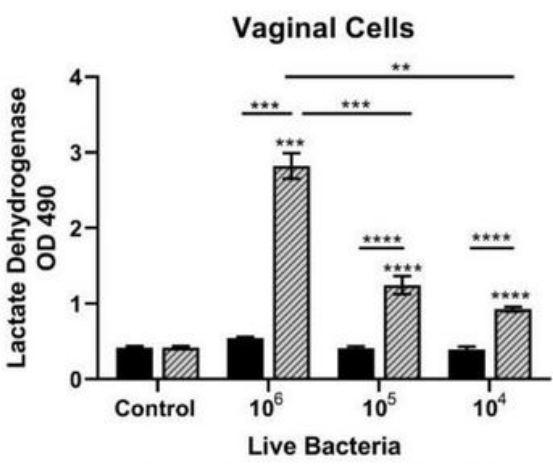

- L. crispatus G. vaginalis

\section{Figure 1}

Co-localization of cervicovaginal epithelial cells with live $G$. vaginalis but not $L$. crispatus results in increased cell death. An in vitro live bacteria and host cervicovaginal co-culture model was created to study host microbial interactions in the CV space. Representative images of ectocervical (A, B), endocervical (C, D) and vaginal (E, F) epithelial cells interacting with $L$. crispatus (A, C, E) or G. vaginalis $(B, D, F)$ are shown. Exposure of ectocervical $(G)$, endocervical $(H)$ and vaginal $(I)$ cells to $G$. vaginalis but not $L$. crispatus results in dose-dependent cell death after 24 hours. Values are mean \pm SEM. Asterisks over the individual bars represent comparisons to control; asterisks over solid lines represent comparisons between treatment groups. ${ }^{*} p<0.05,{ }^{*} p<0.01,{ }^{* \star *} p<0.001,{ }^{* \star \star *} p<0.0001$. 
A.

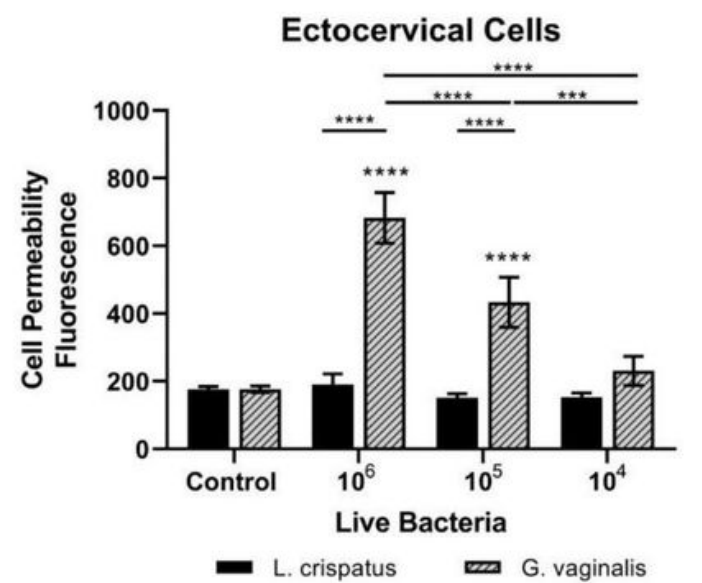

Endocervical Cells

C.

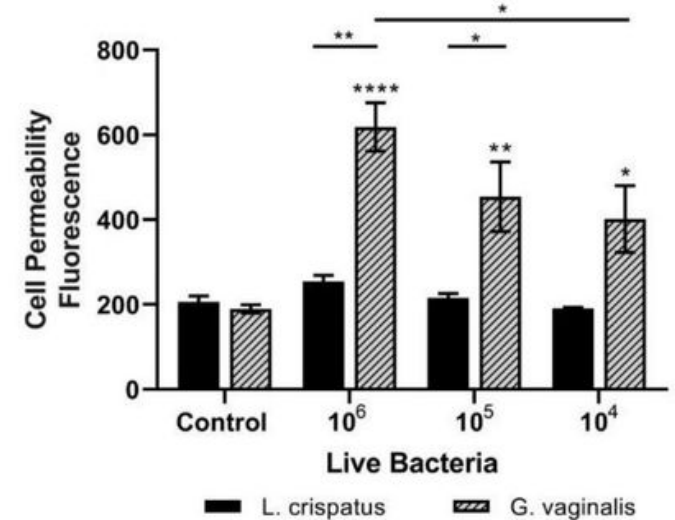

E.

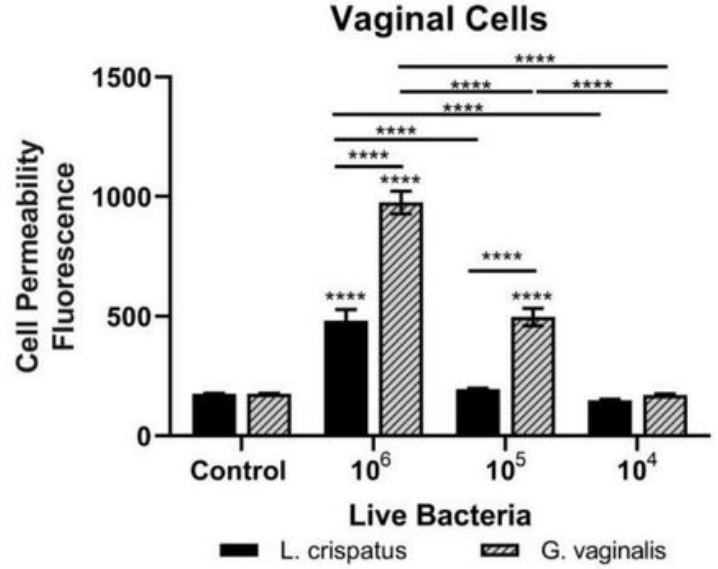

B. Ectocervical Cells

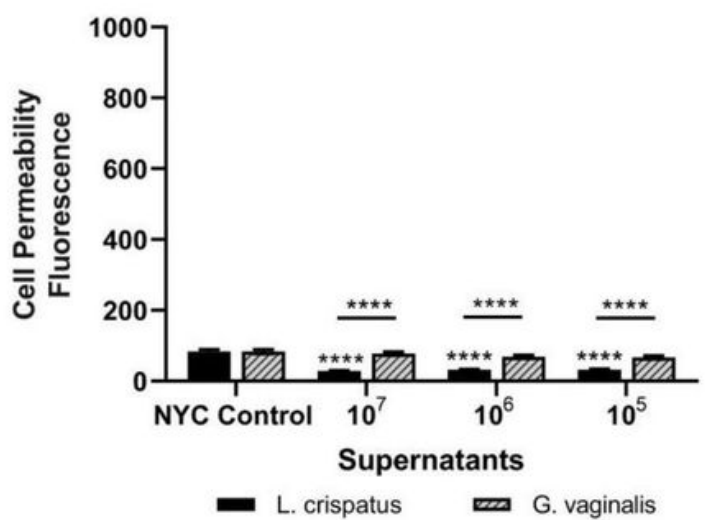

Endocervical Cells

D.

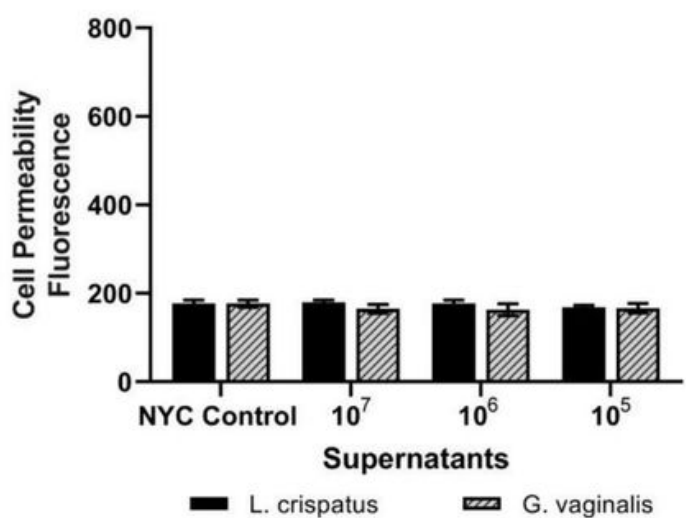

F.

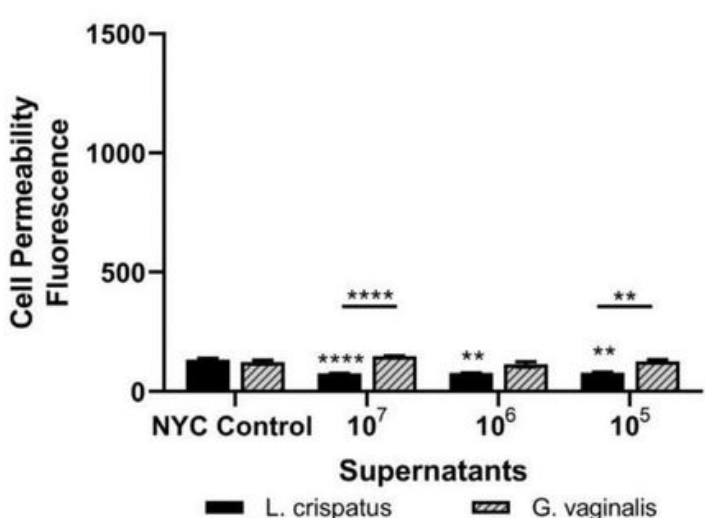

Figure 2

Live G. vaginalis increases epithelial barrier permeability. Cell permeability was measured in ectocervical, endocervical and vaginal epithelial cells after 24-hour exposure to live bacteria (A, C, E) or bacteria-free supernatants (B, D, F) of $L$. crispatus or $G$. vaginalis. Bacterial growth media alone acted as a negative control for the bacteria-free supernatants tested. Cell permeability is expressed as fluorescence OD measurements from a fluorescent plate reader and is indicative of the movement of FITC-dextran from the top to the bottom insert of a transwell chamber system. Values are mean \pm SEM. Asterisks over the 
individual bars represent comparisons to control; asterisks over solid lines represent comparisons between treatment groups. ${ }^{*} p<0.05,{ }^{\star \star} p<0.01,{ }^{\star \star *} p<0.001,{ }^{\star \star \star *} p<0.0001$.

A.

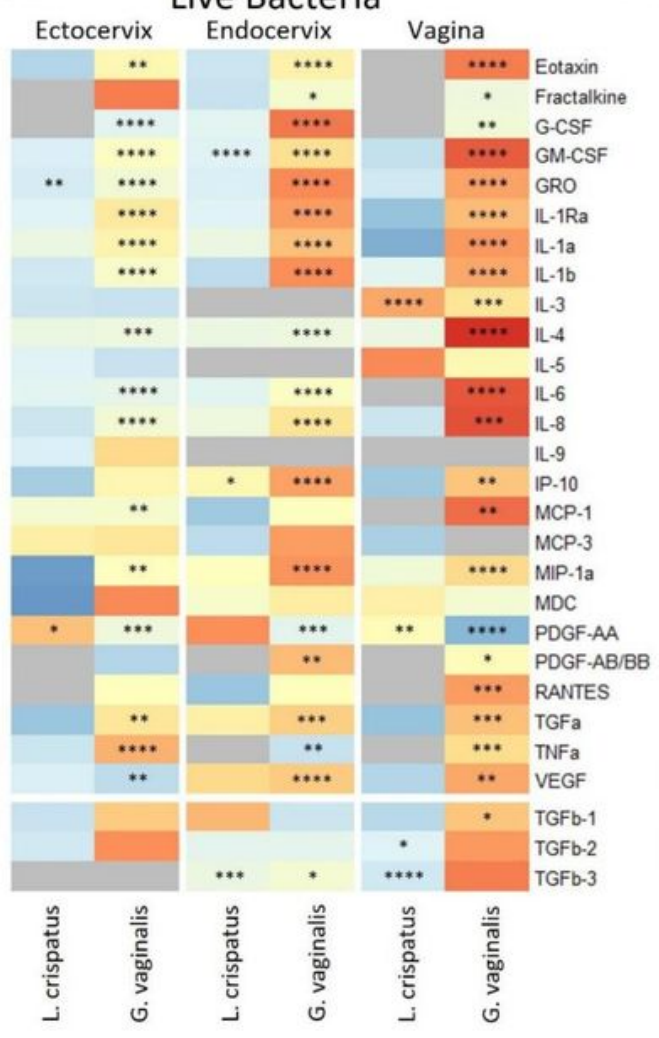

B. Bacterial Supernatant

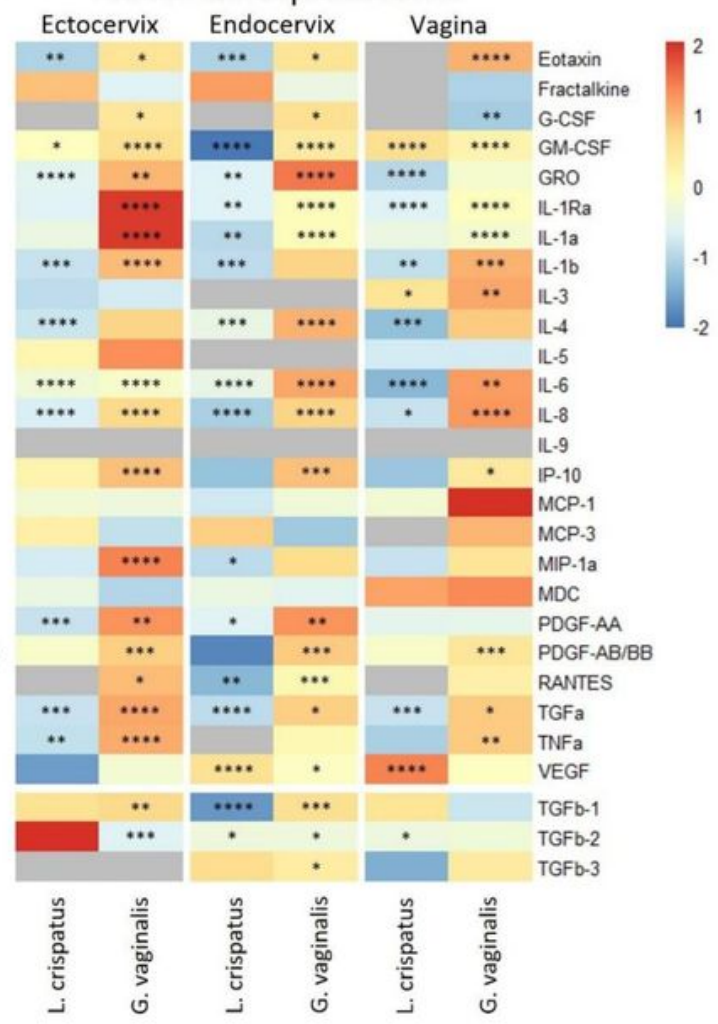

D.
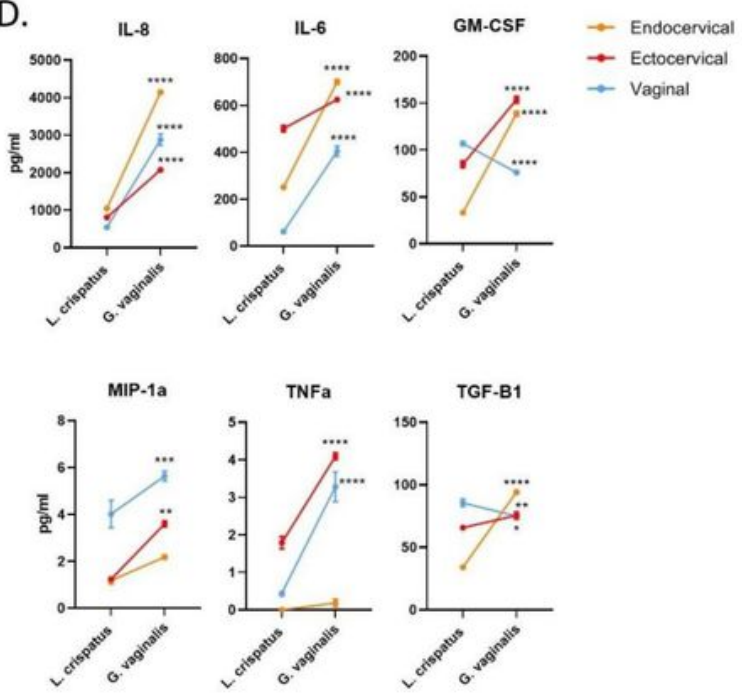

Figure 3

Live $G$. vaginalis activates the hostepithelial immune response while bacteria-free supernatants alter immune activation in a non-bacteria specific manner. Immune cytokines/chemokines released from 
ectocervical, endocervical and vaginal cells after exposure to live L. crispatus and G. vaginalis (A) or their bacteria-free supernatants (B) for 24 hours were measured by Luminex. Heat map depicts fold change (vs NTC for live bacteria or vs NYC for bacteria-free supernatants) by color and p-value by asterisks.

Representative graphs of cytokines $(\mathrm{pg} / \mathrm{mL})$ showing significant differences between L. crispatus and $G$. vaginalis exposure for both live bacteria $(C)$ and bacteria-free supernatants (D) show epithelial cell specific responses. Values are mean \pm SEM. ${ }^{*} p<0.05,{ }^{* \star} p<0.01,{ }^{\star \star *} p<0.001,{ }^{* \star * \star} p<0.0001$.

A.

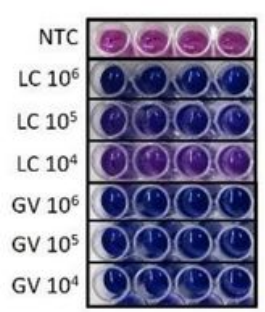

E.

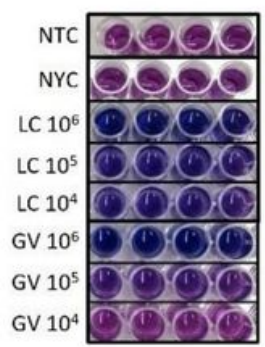

B.

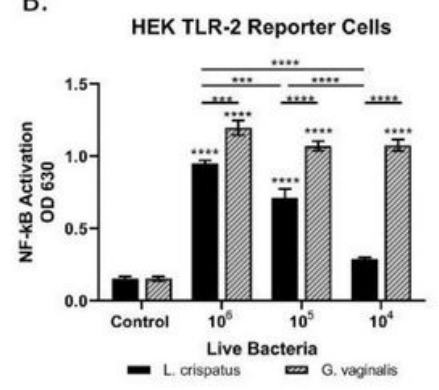

F.

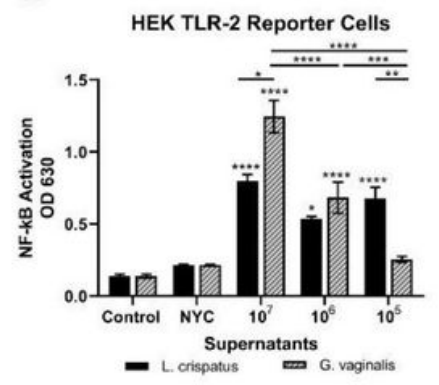

C.

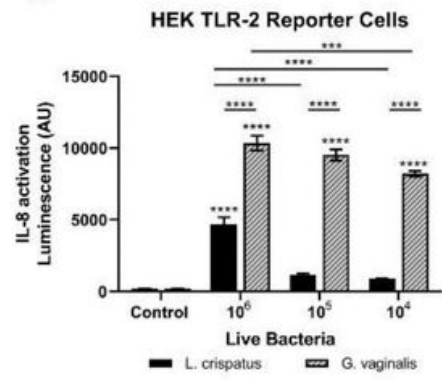

G.

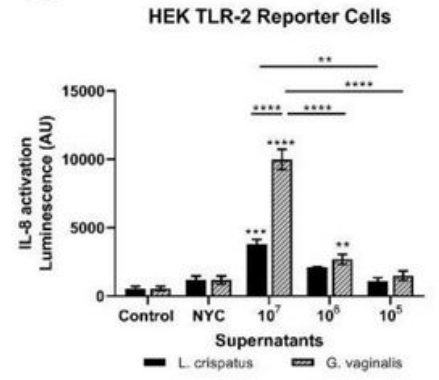

D.

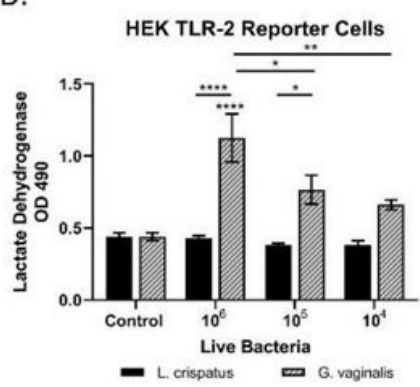

H.

HEK TLR-2 Reporter Cells

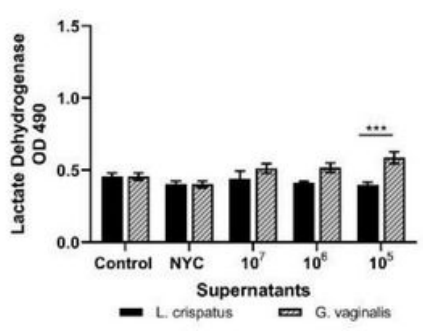

\section{Figure 4}

L. crispatus and G. vaginalis activate NFkB signaling through TLR2, however, only G. vaginalis results in increased IL-8 levels. The HEK TLR2 reporter cell line was used to determine if either live or bacteria-free supernatants from L. crispatus and G. vaginalis activated TLR2-mediated cell signaling. Representative images $(A, E)$ and the corresponding quantification $(B, F)$ of the Quanti-blue NFkB detection assay, IL-8 activation (SEAP quantification) $(C, G)$ and cytotoxicity $(D, H)$ were all altered after exposure to live bacteria and bacteria-free supernatants from $L$. crispatus and $G$. vaginalis. For $A$ and $E$, darker blue/purple indicates higher NFkB. Values are mean \pm SEM. Asterisks over the individual bars represent comparisons to control; asterisks over solid lines represent comparisons between treatment groups. ${ }^{*} p<0.05,{ }^{* *} p<0.01,{ }^{* \star *} p<0.001,{ }^{* \star \star *} p<0.0001$. 
A.

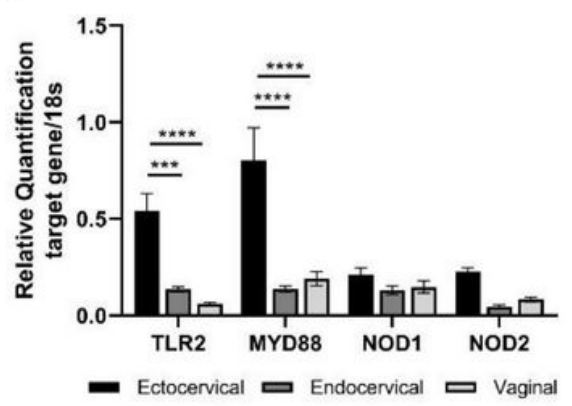

D.

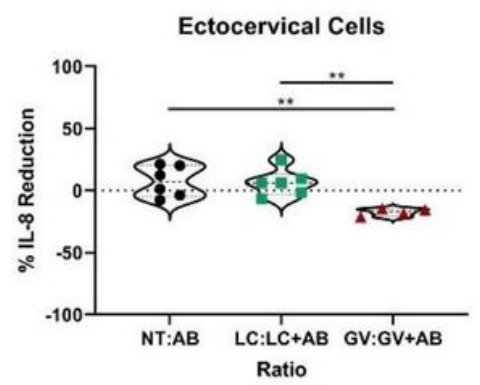

B.

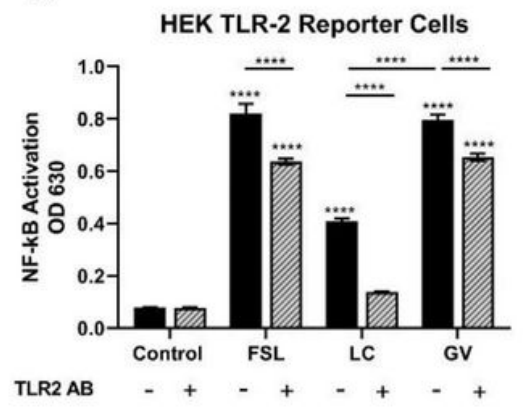

E.

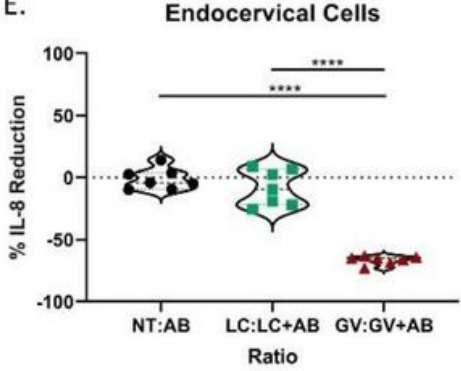

C.

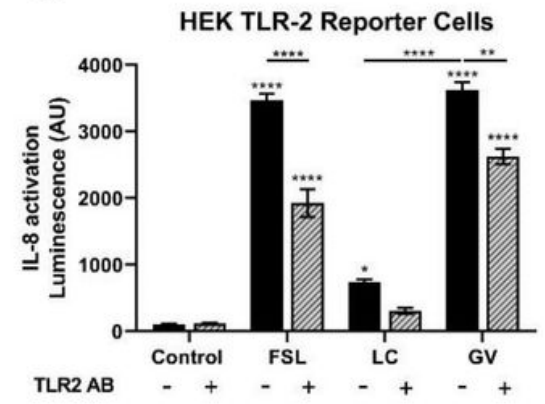

F.

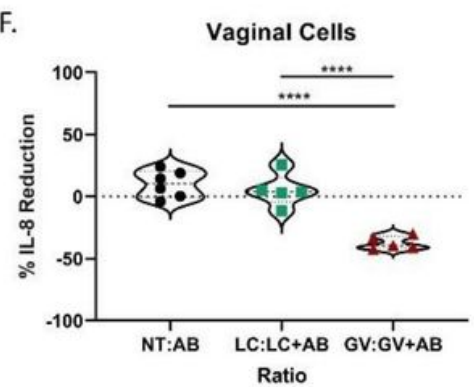

Figure 5

Blocking the TLR2 receptor significantly reduces $L$. crispatus and G. vaginalis-induced NFkB and IL-8 activation. TLR2, MYD88, NOD1 and NOD2 are expressed in cervicovaginal epithelial cells but expression varies by epithelial cell type (A). Blocking the TLR2 receptor in the HEK TLR2 reporter cells significantly reduced L. crispatus and G. vaginalis-induced NFkB activation (B) and only G. vaginalis-induced IL-8 (C). The TLR2 agonist FSL was included as a positive control. Blocking the TLR2 receptor in cervicovaginal epithelial cells resulted in a reduction in G. vaginalis-induced IL-8 but no effect was see in L. crispatus treated cells $(D, E, F)$. Data is expressed as a percent reduction of the ratio of treatment to treatment plus anti-TLR antibody (AB). Values are mean \pm SEM. Asterisks over the individual bars represent comparisons to control; asterisks over solid lines represent comparisons between treatment groups. ${ }^{\star} \mathrm{p}<0.05,{ }^{* \star} \mathrm{p}<0.01$, $\star \star \star * p<0.001, \star \star \star \star x p<0.0001$. 
A.

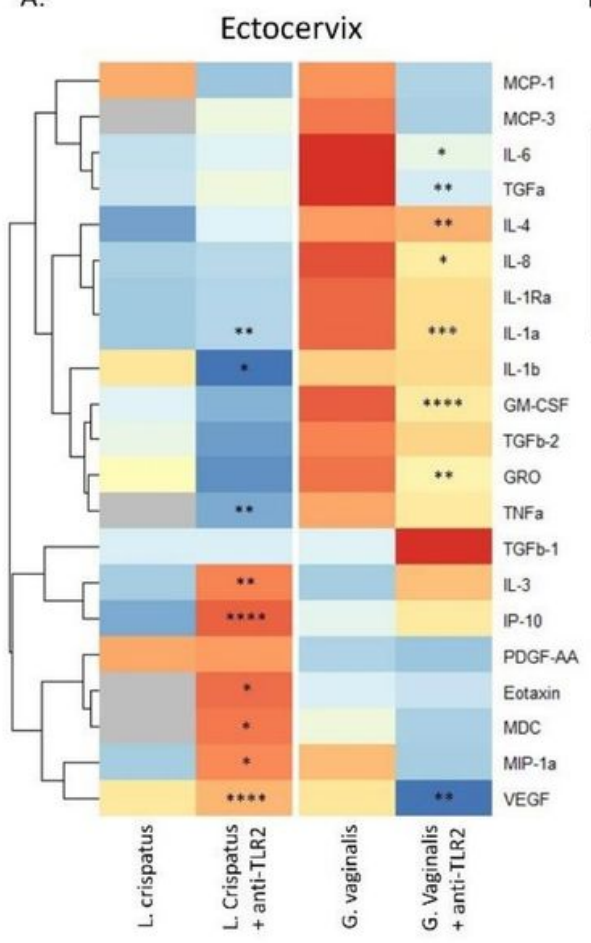

B.

Endocervix

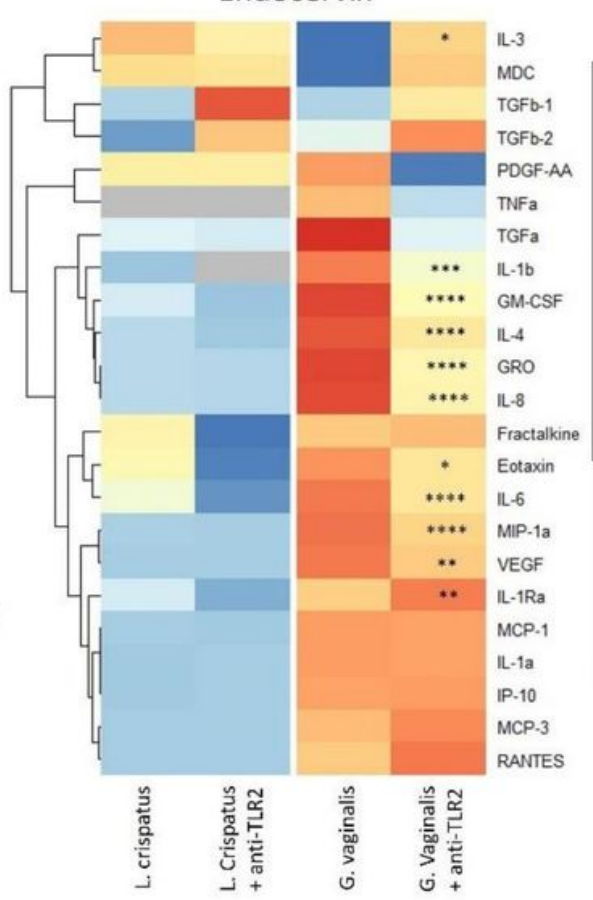

C.

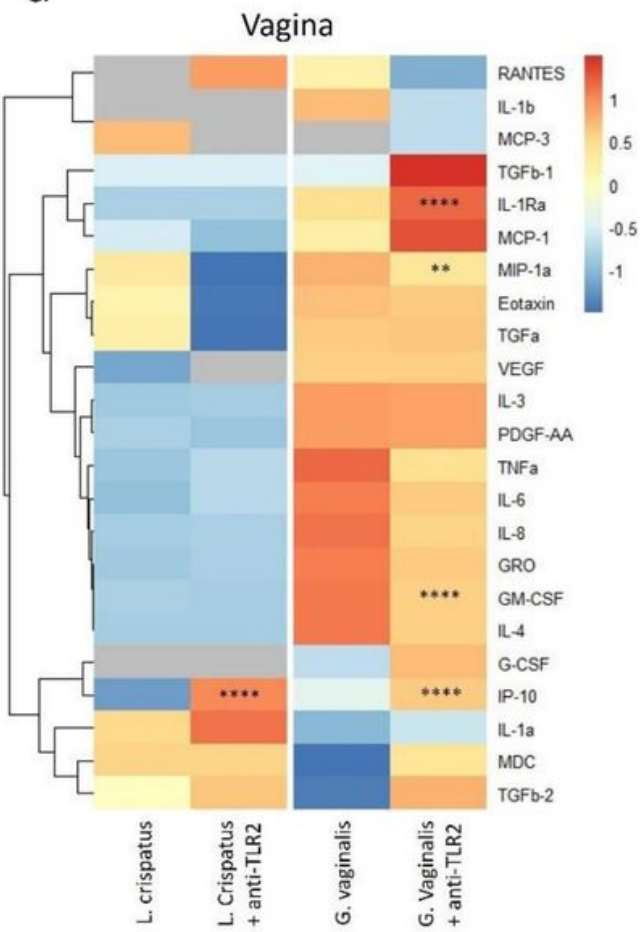

Figure 6

Immune profile of cervicovaginal epithelial cells identifies cytokines mediated by activation of TLR2. Blocking the TLR2 receptor alters the immune cytokines/chemokines released from ectocervical (A), endocervical (B) and vaginal cells (C) after exposure to live L. crispatus and G. vaginalis. Heat map depicts fold change by color and p-value by asterisks. Fold change was calculated between live bacteria and live bacteria plus anti-TLR2 antibody. ${ }^{*} p<0.05,{ }^{* \star} p<0.01,{ }^{* \star *} p<0.001,{ }^{* \star \star *} p<0.0001$.

\section{Figure 7}

Blocking the TLR2 receptor does not mitigate $G$. vaginalis-induced increases in cell permeability. Cell permeability was measured in ectocervical (A), endocervical (B) and vaginal (C) epithelial cells after pretreatment with the anti-TLR2 antibody and subsequent exposure to live bacteria. G. vaginalis-induced cell permeability was unchanged after blocking the TLR2 receptor. Values are mean \pm SEM. 


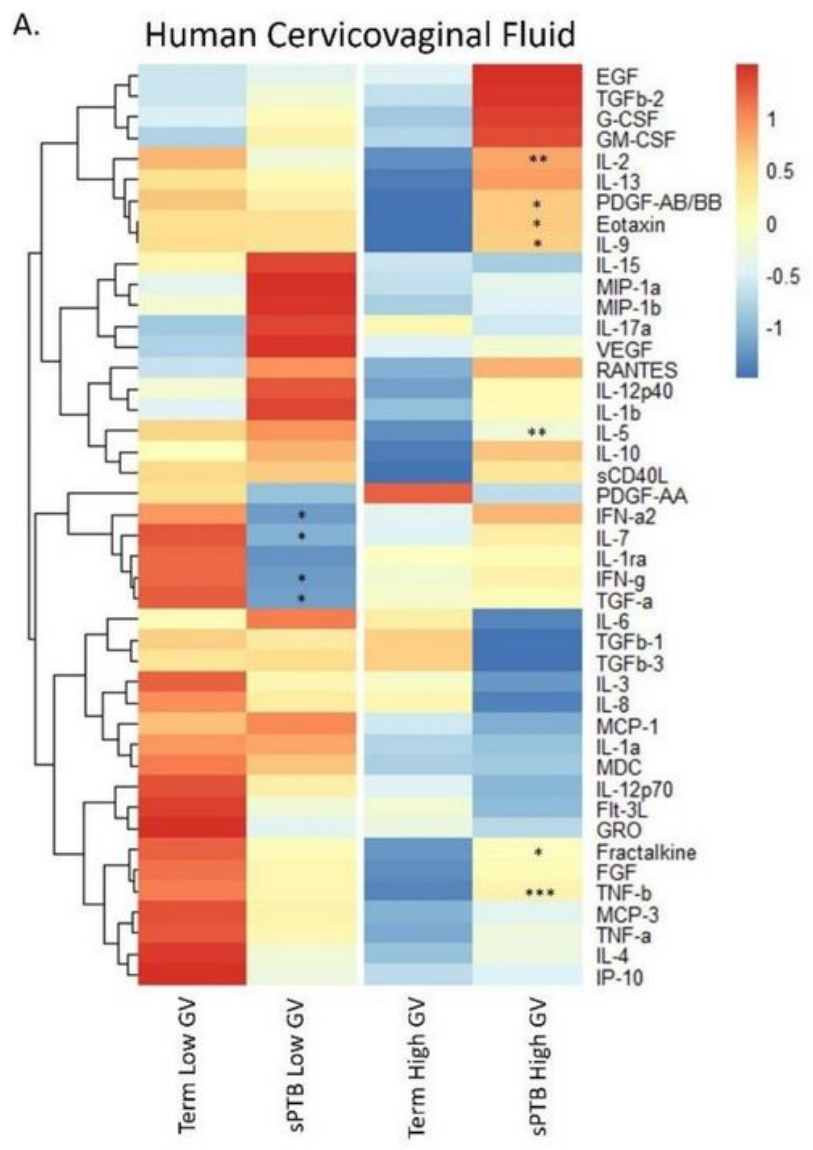

B.

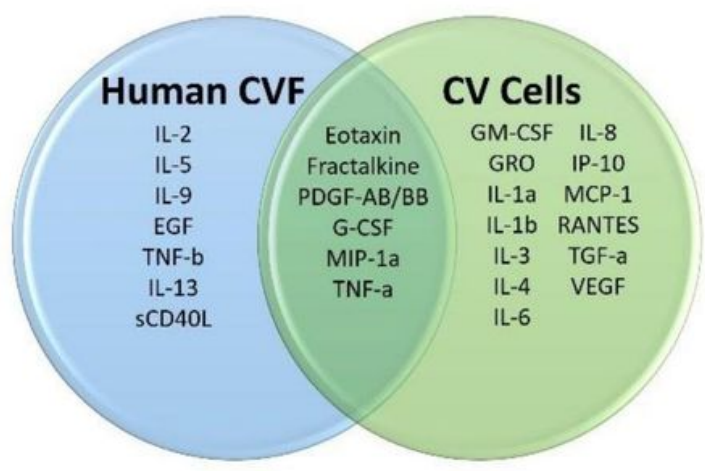

C.
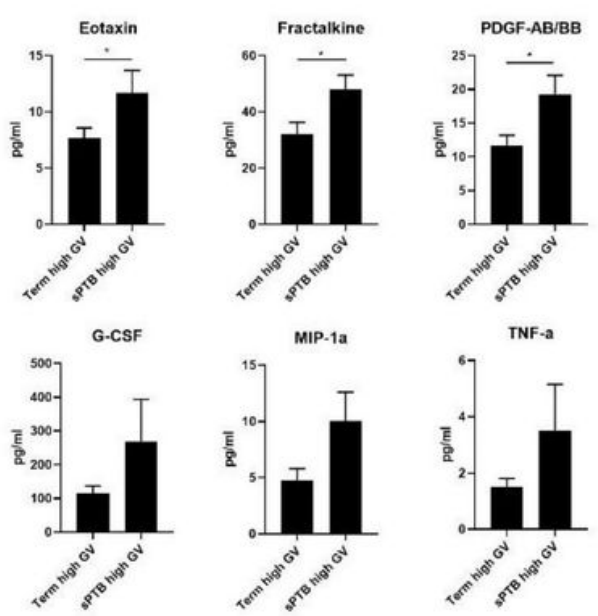

\section{Figure 8}

Immune profile from human cervicovaginal fluid is altered by abundance of $G$. vaginalis and delivery outcome. Cervicovaginal swabs collected from CST IV pregnant individuals at 16-20 weeks of gestation with either term or sPTB deliveries were used for Luminex assays to determine immune profiles of cytokines/chemokines. Heat map shows immune profiles vary by both $G$. vaginalis abundance as well delivery outcome (A). Ven diagram shows overlapping cytokines $(p<0.10)$ induced in CVF from individuals with high $G$. vaginalis abundance and a sPTB versus cervicovaginal epithelial cells exposed to live $G$. vaginalis in culture (B). Graphs of overlapping cytokines are shown in C. Heat map (A) values are based on $\mathrm{pg} / \mathrm{mL}$ and expressed in a relative scale. Values are mean $\pm \mathrm{SEM}$. ${ }^{*} \mathrm{p}<0.05$ and a trend for significance $=p<0.10$.

\section{Supplementary Files}

This is a list of supplementary files associated with this preprint. Click to download.

- AntonL.supplementalinfo01112022.docx 